\title{
THREE MANAGEMENT POLICIES FOR A RESOURCE WITH PARTITION CONSTRAINTS
}

\author{
MURAT ALANYALI, ${ }^{*}$ Bilkent University
}

\begin{abstract}
Management of a bufferless resource is considered under non-homogeneous demand consisting of one-unit and two-unit requests. Two-unit requests can be served only by a given partition of the resource. Three simple admission policies are evaluated with regard to revenue generation. One policy involves no admission control and two policies involve trunk reservation. A limiting regime in which demand and capacity increase in proportion is considered. It is shown that each policy is asymptotically optimal for a certain range of parameters. Limiting dynamical behavior is obtained via a theory developed by Hunt and Kurtz. The results also point out the remarkable effect of partition constraints.
\end{abstract}

Keywords: Resource management; partition constraints; loss networks; multirate networks; admission control; trunk reservation; heavy traffic; time-scale separation

AMS 1991 Subject Classification: Primary 60K30

Secondary 90B22; 68M20; 93E20

\section{Introduction}

This paper investigates effective control policies for a bufferless resource that operates under non-homogeneous dynamic demand. The demand consists of requests of two different types, categorized by the number of resource units required for service. Management of the resource is subject to partition constraints: requests of each type can be serviced only by a block from an associated partition of the resource. We consider in detail the case when one type requires twice as many resource units as the other.

Partition constraints typically arise in time-division-multiplexed multirate communication systems, owing to certain operational limitations. An instance of the problem addressed in this paper arose in the global system for mobile communication (GSM). The system accommodates full-rate users, each of which requires a full-time-slot, as well as half-rate users, each of which requires a half-time-slot. Here a full-time-slot refers to a time-slot in each transmitted frame, and a half-time-slot refers to a time-slot in every other transmitted frame. A pair of half-timeslots can accommodate a full-rate user only if they form a full-time-slot, so the collection of all such pairs constitutes a resource partition for full-rate users.

We consider the following stochastic setting. Let $\lambda_{\mathrm{f}}, \lambda_{\mathrm{h}}$, and $C$ be fixed positive numbers, and let $\gamma$ be a positive scaling factor. There are two types of calls denoted by full-rate calls and half-rate calls. Full-rate calls arrive according to a Poisson process of rate $\gamma \lambda_{\mathrm{f}}$ and half-rate calls arrive according to a Poisson process of rate $\gamma \lambda_{\mathrm{h}}$. The two arrival processes are mutually

\footnotetext{
Received 24 March 1998; revision received 8 September 1998.

* Postal address: Department of Electrical and Electronic Engineering, Bilkent University, Bilkent TR-06533, Ankara, Turkey.

Email address: alanyali@ee.bilkent.edu.tr

This work was supported in part by the US National Science Foundation under contract NSF NCR 93-14253.
} 
independent. The total number of available slots is $\lfloor\gamma C\rfloor$. A slot can be assigned either one full-rate call or at most two half-rate calls. There is no buffering, thus a call is blocked if it is not assigned a slot immediately upon its arrival. Blocked calls cannot be assigned later, and have no effect on the future evolution of the system. A slot is said to be occupied if it is assigned one full-rate call or two half-rate calls, partially occupied if it is assigned one half-rate call, or idle otherwise. A full-rate call is blocked if upon its arrival there are no idle slots, and a half-rate call is blocked if upon its arrival all slots are occupied. Calls can also be blocked in other circumstances depending on the admission policy, which is a decision mechanism to accept or reject an arriving call. For efficient use of capacity, an accepted half-rate call is assigned an idle slot only if there are no partially occupied slots at the time of its arrival. Each accepted call remains in the system for the duration of its holding time, during which it maintains the same slot assignment. The holding time of a call is an exponentially distributed random variable with unit mean, independent of its type and the history of the system prior to its arrival. If accepted, each full-rate call generates revenue at rate $r_{\mathrm{f}}$ and each half-rate call generates revenue at rate $r_{\mathrm{h}}$ throughout the holding time.

A similar stochastic setting in which calls require either one or six resource units has been a subject of considerable interest in the context of ISDN communication systems. In that setting Ramaswami and Rao (1985) studied approximate call blocking probabilities in the absence of admission control. Reiman and Schmitt (1994) considered trunk reservation type admission policies as well, and studied effective methods to determine the blocking probabilities in the case when call types have vastly different time scales. Ross and Tsang (1989) focused on efficient methods to determine admission policies that maximize resource utilization.

In this paper effectiveness of an admission policy is measured with the revenue generated in the long term. We examine three policies which have desirable features such as simplicity and robustness to traffic parameters. These policies are evaluated in a limiting regime that corresponds to arbitrarily large values of the scaling factor $\gamma$, and it is shown that asymptotically each policy generates revenue at maximum rate for certain values of the parameters $\left(r_{\mathrm{f}}, r_{\mathrm{h}}\right)$. In addition to equilibrium properties, explicit descriptions of the transient system behavior are also obtained.

The first policy considered in the paper is trunk reservation for full-rate calls, under which a full-rate call is accepted whenever there is an idle slot, whereas a half-rate call is accepted only if the number of idle slots is larger than a reservation threshold $T(\gamma)$. Note that acceptance of a half-rate call does not depend on the availability of partially occupied slots. The reservation threshold grows unboundedly with $\gamma$ (i.e. $\lim _{\gamma \rightarrow \infty} T(\gamma)=\infty$ ), however slower than $\gamma$ itself (i.e. $\lim _{\gamma \rightarrow \infty} T(\gamma) / \gamma=0$ ). The second policy, trunk reservation for half-rate calls, prescribes accepting a half-rate call unless all slots are occupied, and accepting a full-rate call only if the number of idle slots is larger than $T(\gamma)$. Finally we consider complete sharing under which no admission control is exercised, so that a call is accepted if there is enough capacity to accommodate it.

Trunk reservation has been studied extensively in stochastic settings that do not involve partition constraints. Miller (1969) showed that under homogeneous traffic a trunk reservation policy maximizes the rate of revenue generation among non-anticipative admission policies. If either the request size or the mean holding time varies with call type, such a conclusion holds in a limiting regime similar to the one considered here, as established by Hunt and Laws (1997). The work of Hunt and Laws (1997) is closely related to the work of Bean et al. (1995, 1997) which studies the limiting behavior of trunk reservation. All three papers are based on the theory developed in Hunt and Kurtz (1994) which provides a detailed description of 
the limiting system dynamics, particularly on boundaries along which the system statistics are discontinuous. In the context of the present paper such boundaries arise as a result of depletion of idle or partially occupied slots, and our analysis also is based on Hunt and Kurtz (1994).

In the remainder of this section we state the main results of the paper, starting with some essential definitions. For each $t \geq 0$ let the random vector $X_{t}=\left(X_{t}(1), X_{t}(2), X_{t}(3)\right)$ be defined as

$$
\begin{aligned}
& X_{t}(1)=\text { number of slots occupied by full-rate calls at time } t \\
& X_{t}(2)=\text { number of slots occupied by (two) half-rate calls at time } t \\
& X_{t}(3)=\text { number of partially occupied slots at time } t
\end{aligned}
$$

and set $X_{t}^{\gamma}=\left(X_{t}(1) / \gamma, X_{t}(2) / \gamma, X_{t}(3) / \gamma\right)$. An initial condition and an admission policy determine the random processes $X=\left(X_{t}: t \geq 0\right)$ and $X^{\gamma}=\left(X_{t}^{\gamma}: t \geq 0\right)$. The long-term average rate of revenue generated by an admission policy $\Pi, J_{\Pi}$, is expressed as

$$
J_{\Pi}=\limsup _{T \rightarrow \infty} \mathrm{E}\left[\frac{1}{T} \int_{0}^{T}\left\{r_{\mathrm{f}} X_{t}(1)+r_{\mathrm{h}}\left(2 X_{t}(2)+X_{t}(3)\right)\right\} \mathrm{d} t\right] .
$$

Under each of the three admission policies of interest, the process $X^{\gamma}$ is ergodic and $X_{\infty}^{\gamma}$ denotes the equilibrium random vector. Given real numbers $a, b$ let $a \wedge b$ denote the smaller of $a$ and $b$, and define

$$
\begin{aligned}
& x^{*}=\left(C \wedge \lambda_{\mathrm{f}},\left(C-x^{*}(1)\right) \wedge\left(\lambda_{\mathrm{h}} / 2\right), 0\right) \\
& x_{*}=\left(\left(C-x_{*}(2)\right) \wedge \lambda_{\mathrm{f}}, C \wedge\left(\lambda_{\mathrm{h}} / 2\right), 0\right) .
\end{aligned}
$$

The main contribution of the paper has two aspects. First, asymptotic optimality of the admission policies considered is established by the following three theorems. Here it is remarkable that complete sharing asymptotically achieves full priority for half-rate calls without the need for trunk reservation. Second, a methodical approach is shown to identify the limiting dynamical behavior via the theory of Hunt and Kurtz (1994).

Theorem 1.1. Under trunk reservation for full-rate calls (TRF) $\lim _{\gamma \rightarrow \infty} X_{\infty}^{\gamma}=x^{*}$ in probability. In particular if $r_{\mathrm{f}} \geq 2 r_{\mathrm{h}}$ then for any admission policy $\Pi$

$$
\limsup _{\gamma \rightarrow \infty} J_{\Pi} / \gamma \leq \lim _{\gamma \rightarrow \infty} J_{\mathrm{TRF}} / \gamma=r_{\mathrm{f}} x^{*}(1)+r_{\mathrm{h}}\left(2 x^{*}(2)+x^{*}(3)\right)
$$

Theorem 1.2. Under trunk reservation for half-rate calls $(T R H) \lim _{\gamma \rightarrow \infty} X_{\infty}^{\gamma}=x_{*}$ in probability. In particular if $r_{\mathrm{f}} \leq 2 r_{\mathrm{h}}$ then for any admission policy $\Pi$

$$
\limsup _{\gamma \rightarrow \infty} J_{\Pi} / \gamma \leq \lim _{\gamma \rightarrow \infty} J_{\mathrm{TRH}} / \gamma=r_{\mathrm{f}} x_{*}(1)+r_{\mathrm{h}}\left(2 x_{*}(2)+x_{*}(3)\right) \text {. }
$$

Theorem 1.3. Under complete sharing $(C S) \lim _{\gamma \rightarrow \infty} X_{\infty}^{\gamma}=x_{*}$ in probability. In particular if $r_{\mathrm{f}} \leq 2 r_{\mathrm{h}}$ then for any admission policy $\Pi$

$$
\limsup _{\gamma \rightarrow \infty} J_{\Pi} / \gamma \leq \lim _{\gamma \rightarrow \infty} J_{\mathrm{CS}} / \gamma=r_{\mathrm{f}} x_{*}(1)+r_{\mathrm{h}}\left(2 x_{*}(2)+x_{*}(3)\right) \text {. }
$$


(a)

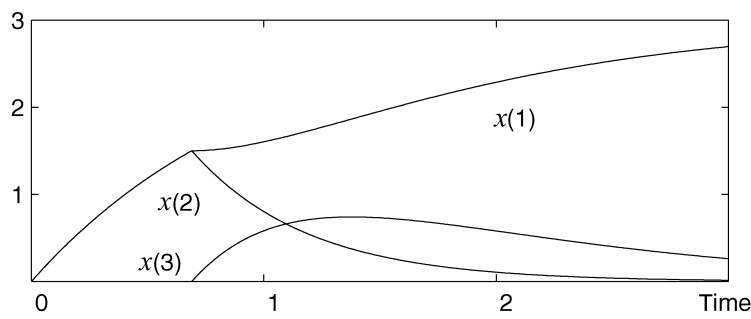

(b)

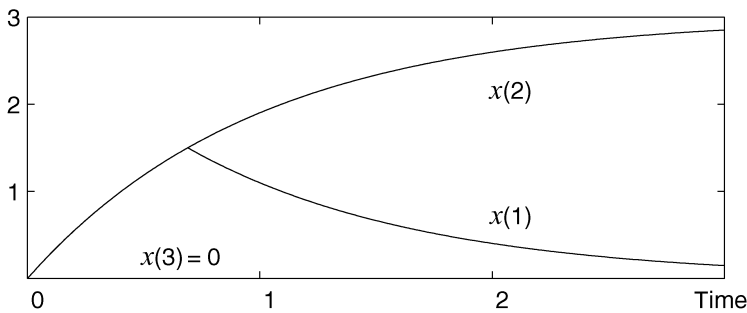

(c)

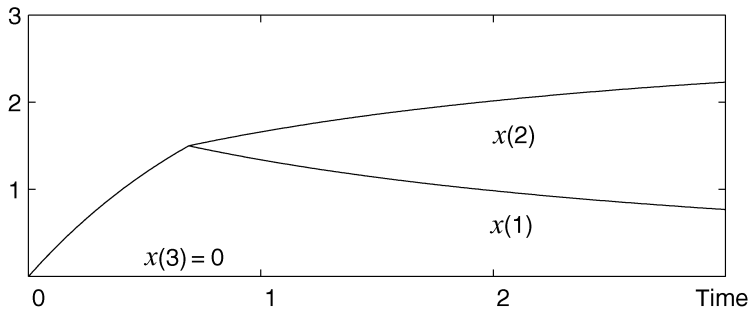

FIGURE 1: Typical trajectories that approximate the transient behavior of the system under (a) trunk reservation for full-rate calls, (b) trunk reservation for half-rate calls, and (c) complete sharing, in the case $C=\lambda_{\mathrm{f}}=\lambda_{\mathrm{h}} / 2=3$.

We now briefly comment on the theorems. The vector $x^{*}$ (respectively the vector $x_{*}$ ) characterizes an operating point at which the available capacity is used primarily to accommodate full-rate (half-rate) calls, leaving only the excess capacity for half-rate (full-rate) calls. Moreover half-rate calls are almost perfectly packed so that there is only a marginal number of partially occupied slots. If $r_{\mathrm{f}} \geq 2 r_{\mathrm{h}}\left(r_{\mathrm{f}} \leq 2 r_{\mathrm{h}}\right)$ then such an operating point is almost optimal, and by Theorem 1.1 (Theorem 1.2) trunk reservation achieves asymptotic optimality by maintaining the system sufficiently close to it. By Theorem 1.3 the uncontrolled system tends to evolve around the same operating point as the system under the TRH policy, so that complete sharing is also asymptotically optimal if $r_{\mathrm{f}} \leq 2 r_{\mathrm{h}}$.

The partition constraint has a remarkable effect on the natural evolution of the system, as pointed out by Theorem 1.3: in the absence of partition constraints, it follows from Kelly (1986) that complete sharing results in limiting blocking probabilities of $\left(1-q^{2}\right)_{+}$and $(1-q)_{+}$for full-rate and half-rate calls respectively, where $q$ denotes the positive root of $\lambda_{\mathrm{f}} q^{2}+\left(\lambda_{\mathrm{h}} / 2\right) q-C=0$ and $(\cdot)_{+}$denotes $\max (\cdot, 0)$. When the partition constraint is imposed, however, full-rate calls may experience a disproportionately large blocking probability, to the extent that they may be totally locked out of the system in the large $\gamma$ limit.

We finally comment on the transient behavior of the system under the three admission policies. Figure 1 illustrates trajectories that well-approximate the process $X^{\gamma}$ for large values of $\gamma$, in the case $C=\lambda_{\mathrm{f}}=\lambda_{\mathrm{h}} / 2=3$ and $X_{0}^{\gamma}=0$. An intuitive interpretation of these 
trajectories is as follows. As long as idle slots are abundant all arrivals are accepted, in turn the numbers of full-rate and half-rate calls increase exponentially towards $\gamma \lambda_{\mathrm{f}}$ and $\gamma \lambda_{\mathrm{h}}$ respectively. In this regime, assigning half-rate arrivals to partially occupied slots suffices to keep the number of partially occupied slots at $o(\gamma)$, therefore half-rate calls are almost perfectly packed into occupied slots. Since $C<\lambda_{\mathrm{f}}+\left(\lambda_{\mathrm{h}} / 2\right)$, however, the system eventually becomes overloaded. While the system is running at capacity, trunk reservation prioritizes one type of arrivals over the other type; idle capacity generated by departures is typically used to accommodate high priority arrivals. Under trunk reservation for half-rate calls, each full-rate departure immediately enables admission of two half-rate arrivals, in turn half-rate calls experience virtually no blocking. In contrast, under trunk reservation for full-rate calls, a fraction of half-rate departures contribute to the number of partially occupied slots, thereby increasing the number of such slots to $O(\gamma)$ and causing temporary blocking of full-rate arrivals. Under complete sharing, full-rate and half-rate arrivals compete for idle slots. Since the number of partially occupied slots is marginal, half-rate calls release idle slots at a much smaller rate than full-rate calls do; in turn half-rate calls have an inherent advantage. This advantage is significant enough so that eventually half-rate calls monopolize the entire system.

The following three sections provide analyses of the three admission policies, and prove Theorems 1.1, 1.2, and 1.3 respectively. Proofs of some auxiliary results are collected in the Appendix.

\section{Trunk reservation for full-rate calls}

Under trunk reservation for full-rate calls $X^{\gamma}$ is a Markov process that takes values in the state space $S=\left\{z \in \mathbb{R}_{+}^{3}: z(1)+z(2)+z(3) \leq C\right\}$. For each $t \geq 0$ let $F_{t}=\lfloor\gamma C\rfloor-\left(X_{t}(1)+\right.$ $\left.X_{t}(2)+X_{t}(3)\right)$ denote the number of idle slots at time $t$, and set $G_{t}=F_{t}-T(\gamma)$. In the rest of the paper we assume without loss of generality that $T(\gamma)$ takes integer values. Note that at time $t$ a full-rate arrival is accepted if $F_{t^{-}}>0$, whereas a half-rate arrival is accepted if $G_{t^{-}}>0$, in which case it is assigned an idle slot if and only if $X_{t^{-}}(3)=0$. Examination of the generator of $X^{\gamma}$ and Proposition 4.1.7 of Ethier and Kurtz (1986) lead to the following representation:

$$
\begin{aligned}
X_{t}^{\gamma}(1)= & X_{0}^{\gamma}(1)+\lambda_{\mathrm{f}} \int_{0}^{t} \mathbf{1}\left\{F_{s}>0\right\} \mathrm{d} s-\int_{0}^{t} X_{s}^{\gamma}(1) \mathrm{d} s+M_{t}^{\gamma}(1), \\
X_{t}^{\gamma}(2)= & X_{0}^{\gamma}(2)+\lambda_{\mathrm{h}} \int_{0}^{t} \mathbf{1}\left\{X_{s}(3)>0, G_{s}>0\right\} \mathrm{d} s-\int_{0}^{t} 2 X_{s}^{\gamma}(2) \mathrm{d} s+M_{t}^{\gamma}(2), \\
X_{t}^{\gamma}(3)= & X_{0}^{\gamma}(3)+\lambda_{\mathrm{h}} \int_{0}^{t} \mathbf{1}\left\{X_{s}(3)=0, G_{s}>0\right\} \mathrm{d} s+\int_{0}^{t} 2 X_{s}^{\gamma}(2) \mathrm{d} s \\
& -\lambda_{\mathrm{h}} \int_{0}^{t} \mathbf{1}\left\{X_{s}(3)>0, G_{s}>0\right\} \mathrm{d} s-\int_{0}^{t} X_{s}^{\gamma}(3) \mathrm{d} s+M_{t}^{\gamma}(3),
\end{aligned}
$$

for $t \geq 0$, where $\mathbf{1}\{\cdot\}$ denotes the indicator function and the process $M^{\gamma}=\left(M_{t}^{\gamma}: t \geq 0\right)$ is a martingale such that $M_{0}^{\gamma}=0$.

This section proves Theorem 1.1 on the asymptotic optimality of trunk reservation for fullrate calls in the case $r_{\mathrm{f}} \geq 2 r_{\mathrm{h}}$. The outline of the proof is as follows. In Section 2.1 the sequence $\left(X^{\gamma}: \gamma>0\right)$ is shown to be tight in the Skorohod space $D_{\mathbb{R}_{+}^{3}}[0, \infty)$ of right-continuous functions with left limits. The general form of the limits of convergent subsequences is also identified. This form involves multi-valued mappings, and it is refined in Section 2.2 
which establishes that the limit trajectories conform to certain ordinary differential equations in various regions of the state space. Finally Section 2.3 shows that each such trajectory converges to the point $x^{*}$, which in turn leads to the proof of the theorem.

\subsection{Convergence}

This section establishes existence and characterization of weak limits of the sequence $\left(X^{\gamma}: \gamma>0\right)$. The discussion is based on an adaptation of the theory developed in Hunt and Kurtz (1994), which leads to a representation of a weak limit in terms of certain ergodic properties of an auxiliary process. The reader is urged to read the paper of Hunt and Kurtz (1994) in order to better understand the method used here. We start with some essential definitions.

Let $\mathbb{Z}$ and $\mathbb{Z}_{+}$denote the set of integers and the set of non-negative integers respectively. Let $\mathbb{Z}_{+}^{\Delta}=\mathbb{Z}_{+} \cup\{+\infty\}$ and $\mathbb{Z}^{\Delta}=\mathbb{Z}_{+} \cup\{+\infty,-\infty\}$, and set $E=\mathbb{Z}_{+}^{\Delta} \times \mathbb{Z}_{+}^{\Delta} \times \mathbb{Z}^{\Delta}$. For each $y \in E$ define $f(y)=(\tanh (y(1)), \tanh (y(2)), \tanh (y(3)))$, with the understanding that $\tanh ( \pm \infty)= \pm 1$. Endow $E$ with the metric induced from the Euclidean metric on $\mathbb{R}^{3}$ by the mapping $f: E \mapsto \mathbb{R}^{3}$, so that $E$ is compact. Represent by $\mathcal{B}(E)$ the Borel subsets of $E$. Let $\mathcal{L}_{0}(E)$ denote the space of Borel measures $\mu$ on the product space $[0, \infty) \times E$ such that $\mu([0, t) \times E)=t$ for $t \geq 0$. Endow $\mathcal{L}_{0}(E)$ with the topology of weak convergence of measures restricted to $[0, t) \times E$ for each $t \geq 0$. Since $E$ is compact, $\mathcal{L}_{0}(E)$ is compact by Prohorov's theorem.

Define the sets $A_{1}=\{y \in E: y(2)>0\}, A_{2}=\{y \in E: y(1)>0, y(3)>0\}$, and $A_{3}=\{y \in E: y(1)=0, y(3)>0\}$ with the understanding that $-\infty<k<+\infty$ for all $k \in \mathbb{Z}$. Let the feedback process $V=\left(V_{t}: t \geq 0\right)$ be defined by setting $V_{t}=\left(X_{t}(3), F_{t}, G_{t}\right)$ for each $t \geq 0$. Note that the admission and allocation decisions are based on the feedback process, in that, at time $t>0$ a full-rate call is accepted if $V_{t^{-}} \in A_{1}$, and a half-rate call is accepted if $V_{t^{-}} \in A_{2} \cup A_{3}$, in which case it is assigned an idle slot if and only if $V_{t^{-}} \in A_{3}$. Let the random measure $v^{\gamma} \in \mathcal{L}_{0}(E)$ be defined by

$$
v^{\gamma}([0, t) \times B)=\int_{0}^{t} \mathbf{1}\left\{V_{s} \in B\right\} \mathrm{d} s, \quad t \geq 0, \quad B \in \mathcal{B}(E),
$$

so that for $t \geq 0$

$$
\begin{gathered}
X_{t}^{\gamma}(1)=X_{0}^{\gamma}(1)+\lambda_{\mathrm{f}} v^{\gamma}\left([0, t) \times A_{1}\right)-\int_{0}^{t} X_{s}^{\gamma}(1) \mathrm{d} s+M_{t}^{\gamma}(1) \\
X_{t}^{\gamma}(2)=X_{0}^{\gamma}(2)+\lambda_{\mathrm{h}} v^{\gamma}\left([0, t) \times A_{2}\right)-\int_{0}^{t} 2 X_{s}^{\gamma}(2) \mathrm{d} s+M_{t}^{\gamma}(2) \\
X_{t}^{\gamma}(3)=X_{0}^{\gamma}(3)+\lambda_{\mathrm{h}} v^{\gamma}\left([0, t) \times A_{3}\right)+\int_{0}^{t} 2 X_{s}^{\gamma}(2) \mathrm{d} s-\lambda_{\mathrm{h}} v^{\gamma}\left([0, t) \times A_{2}\right) \\
-\int_{0}^{t} X_{s}^{\gamma}(3) \mathrm{d} s+M_{t}^{\gamma}(3) .
\end{gathered}
$$

The compactness of $S$ and $\mathcal{L}_{0}(E)$ imply via Prohorov's theorem tightness of the sequences $\left(X_{0}^{\gamma}: \gamma>0\right)$ and $\left(\nu^{\gamma}: \gamma>0\right)$ respectively. Since $S$ is bounded, the sequence $\left(\int_{0}^{\cdot} X_{s}^{\gamma} \mathrm{d} s\right.$ : $\gamma>0)$ is uniformly equicontinuous, and therefore tight in $D_{\mathbb{R}_{+}^{3}}[0, \infty)$ by Corollary 3.7 .4 of Ethier and Kurtz (1986). Finally Doob's inequality implies that the sequence $\left(M^{\gamma}: \gamma>0\right)$ converges weakly to zero, and it thus follows that $\left(X^{\gamma}: \gamma>0\right)$ is tight in $D_{\mathbb{R}_{+}^{3}}[0, \infty)$, and $\left(\left(X^{\gamma}, v^{\gamma}\right): \gamma>0\right)$ is tight in the associated product space. 


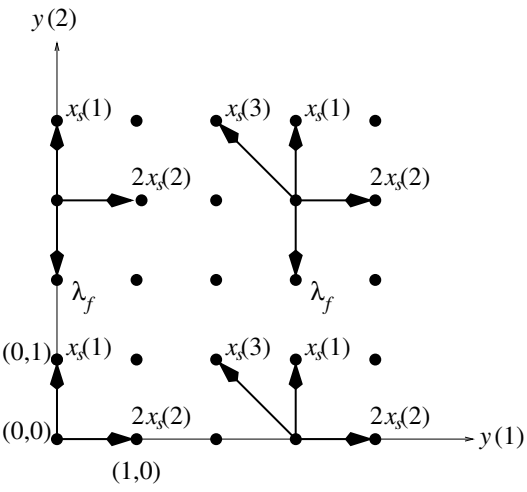

(a)

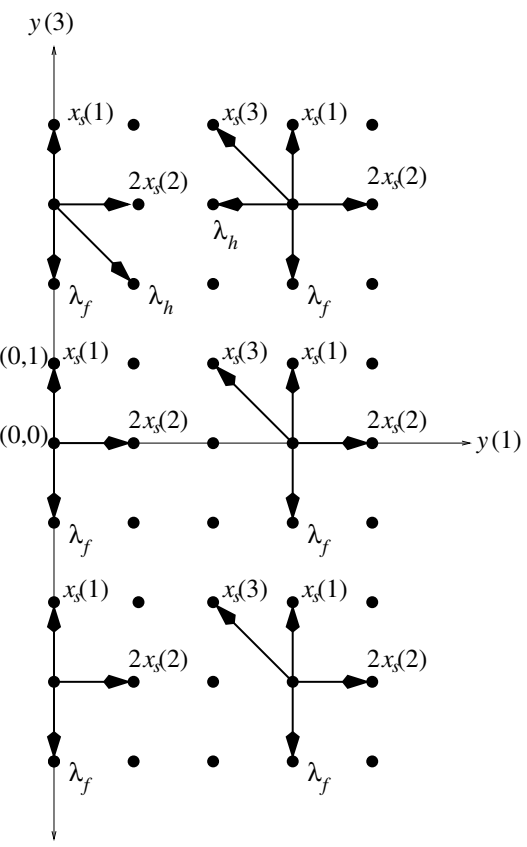

(b)

FIgURE 2: Transition diagrams of (a) the process $\left(Y^{x_{s}}(1), Y^{x_{s}}(2),-\infty\right)$ and (b) the process $\left(Y^{x_{s}}(1)\right.$, $\left.\infty, Y^{x_{s}}(3)\right)$, in the discussion of trunk reservation for full-rate calls.

We now characterize the weak limits of the sequence $\left(\left(X^{\gamma}, v^{\gamma}\right): \gamma>0\right)$ along convergent subsequences. Let $(x, v)$ denote such a limit, and consider first the characterization of the measure $v$. Straightforward adaptation of Theorem 3 of Hunt and Kurtz (1994) yields that $v$ satisfies

$$
v([0, t) \times B)=\int_{0}^{t} \pi_{x_{s}}(B) \mathrm{d} s, \quad t \geq 0, \quad B \in \mathcal{B}(E),
$$

where $\pi_{x_{s}}$ is an equilibrium distribution for a Markov process $Y^{x_{s}}=\left(Y_{t}^{x_{s}}: t \geq 0\right)$ that takes values in $E$ and has transition rates given by

$$
Y^{x_{s}} \leftarrow \begin{cases}Y^{x_{s}}+(0,-1,-1) & \text { at rate } \lambda_{\mathrm{f}} \mathbf{1}\left\{Y^{x_{s}} \in A_{1}\right\} \\ Y^{x_{s}}+(0,+1,+1) & \text { at rate } x_{s}(1) \\ Y^{x_{s}}+(-1,0,0) & \text { at rate } \lambda_{\mathrm{h}} \mathbf{1}\left\{Y^{x_{s}} \in A_{2}\right\} \\ Y^{x_{s}}+(+1,0,0) & \text { at rate } 2 x_{s}(2) \\ Y^{x_{s}}+(+1,-1,-1) & \text { at rate } \lambda_{\mathrm{h}} \mathbf{1}\left\{Y^{x_{s}} \in A_{3}\right\} \\ Y^{x_{s}}+(-1,+1,+1) & \text { at rate } x_{s}(3) \mathbf{1}\left\{Y^{x_{s}}(1)>0\right\} .\end{cases}
$$

Here and in the rest of the paper it is understood that $\pm \infty+k= \pm \infty$ for all $k \in \mathbb{Z}$. In particular $\left(Y^{x_{s}}(1), Y^{x_{s}}(2),-\infty\right)$ and $\left(Y^{x_{s}}(1), \infty, Y^{x_{s}}(3)\right)$ are effectively two-dimensional Markov processes whose transition diagrams are illustrated by Figures 2(a) and 2(b) respectively.

The process $Y^{x_{s}}$ is reducible due to the isolated states at infinity; in turn it admits multiple equilibrium distributions. The distribution $\pi_{x_{s}}$ is therefore some convex combination of the 
equilibrium distributions of $Y^{x_{s}}$ restricted to ergodic closed subsets of the state space. More formally, we adopt the following convention to represent $\pi_{x_{s}}$. Given $i \in E$ let $Y^{x_{s}, i}$ denote the process $Y^{x_{s}}$ conditioned on the initial state $Y_{0}^{x_{s}}=i$, and let $\pi_{x_{s}}^{i}$ denote the unique equilibrium distribution of $Y^{x_{s}, i}$ provided that $Y^{x_{s}, i}$ is ergodic, or an arbitrary distribution otherwise. Then

$$
\pi_{x_{s}}=\sum_{i \in E} p_{x_{s}}(i) \pi_{x_{s}}^{i}
$$

for some probability vector $p_{x_{s}} \in \mathbb{R}_{+}^{E}$ such that $p_{x_{s}}(i)>0$ only if the process $Y^{x_{s}, i}$ is ergodic.

The collection $\left(\pi_{x_{s}}: s \geq 0\right)$ further has the properties asserted by the following lemma. The lemma is proved in the Appendix.

Lemma 2.1. The following conditions hold for almost all $s \geq 0$ :

(a) $\pi_{x_{s}}(y(2)<+\infty, y(3)>-\infty)=0$,

(b) if $x_{s}(3)>0$ then $\pi_{x_{s}}(y(1)=+\infty)=1$,

(c) if $x_{S}(1)+x_{s}(2)+x_{s}(3)<C$ then $\pi_{x_{s}}(y(2)=+\infty, y(3)=+\infty)=1$.

We now provide a characterization of the limit trajectory $x$. For each $j \in\{1,2,3\}$ the function $y \mapsto \mathbf{1}\left\{y \in A_{j}\right\}: E \mapsto\{0,1\}$ is continuous; therefore by the continuous mapping theorem $v^{\gamma}\left([0, t) \times A_{j}\right)$ converges weakly to $v\left([0, t) \times A_{j}\right)$ for each $t \geq 0$. Appeal to Skorohod's Theorem to construct the processes on the same probability space so that the convergence occurs along almost all sample paths. By Theorem 3.10.2 and Lemma 3.10.1 of Ethier and Kurtz (1986) $x$ is continuous and the convergence of $\left(X^{\gamma}: \gamma>0\right)$ is uniform on compact sets; it thus follows that for $t \geq 0$

$$
\begin{aligned}
x_{t} & \in S, \\
x_{t}(1) & =x_{0}(1)+\lambda_{\mathrm{f}} \int_{0}^{t} \pi_{x_{s}}\left(A_{1}\right) \mathrm{d} s-\int_{0}^{t} x_{s}(1) \mathrm{d} s, \\
x_{t}(2) & =x_{0}(2)+\lambda_{\mathrm{h}} \int_{0}^{t} \pi_{x_{s}}\left(A_{2}\right) \mathrm{d} s-\int_{0}^{t} 2 x_{s}(2) \mathrm{d} s, \\
x_{t}(3) & =x_{0}(3)+\lambda_{\mathrm{h}} \int_{0}^{t} \pi_{x_{s}}\left(A_{3}\right) \mathrm{d} s+\int_{0}^{t} 2 x_{s}(2) \mathrm{d} s-\lambda_{\mathrm{h}} \int_{0}^{t} \pi_{x_{s}}\left(A_{2}\right) \mathrm{d} s-\int_{0}^{t} x_{s}(3) \mathrm{d} s .
\end{aligned}
$$

An intuitive interpretation of the above description is as follows. For large values of $\gamma$, the normalized system process $X^{\gamma}=X / \gamma$ is almost constant over small time intervals. In contrast, within such intervals the feedback process takes on many different values due to the large number of arrivals and departures. In the large $\gamma$ limit, the time-scales of the two processes separate; the feedback process reaches equilibrium before the system process changes its value. In particular the instantaneous rates of various admission and allocation decisions at time $s$ are determined by the equilibrium properties of the process $Y^{x_{s}}$ which approximates the localized feedback process $\left(V_{s+(t / \gamma)}: t \in[0, o(\gamma))\right)$.

Some of the general ideas used above have analogues in recent work. Hunt and Laws (1997) employed a construction similar to the feedback process to analyse a trunk reservation policy. An analogue of part ( $a$ ) of Lemma 2.1 is implicit in Hunt and Laws (1997), and parts $(b)$ and (c) of the same lemma follow by straightforward interpretation of Hunt and Kurtz (1994). 
TABLE 1: Valid expressions for $\dot{x}_{t}^{i}$, under trunk reservation for full-rate calls.

\begin{tabular}{lccc}
\hline & $\dot{x}_{t}^{i}(1)$ & $\dot{x}_{t}^{i}(2)$ & $\dot{x}_{t}^{i}(3)$ \\
\hline$i=(+\infty,+\infty,+\infty)$ & $\lambda_{\mathrm{f}}-x_{t}(1)$ & $\lambda_{\mathrm{h}}-2 x_{t}(2)$ & $2 x_{t}(2)-\lambda_{\mathrm{h}}-x_{t}(3)$ \\
$i \in\{+\infty\} \times\{+\infty\} \times \mathbb{Z}$ & Arbitrary & Arbitrary & Arbitrary \\
$i=(+\infty,+\infty,-\infty)$ & $\lambda_{\mathrm{f}}-x_{t}(1)$ & $-2 x_{t}(2)$ & $2 x_{t}(2)-x_{t}(3)$ \\
$i \in\{+\infty\} \times \mathbb{Z}+\times\{-\infty\}$ & $x_{t}(3)$ & $-2 x_{t}(2)$ & $2 x_{t}(2)-x_{t}(3)$ \\
$i \in \mathbb{Z}_{+} \times\{+\infty\} \times\{+\infty\}$ & $\lambda_{\mathrm{f}}-x_{t}(1)$ & $\left(\lambda_{\mathrm{h}} / 2\right)-x_{t}(2)$ & 0 \\
$i \in \mathbb{Z}_{+} \times\{+\infty\} \times \mathbb{Z}$ & $\lambda_{\mathrm{f}}-x_{t}(1)$ & $x_{t}(1)-\lambda_{\mathrm{f}}$ & 0 \\
$i \in \mathbb{Z}_{+} \times\{+\infty\} \times\{-\infty\}$ & $\lambda_{\mathrm{f}}-x_{t}(1)$ & $-2 x_{t}(2)$ & $2 x_{t}(2)$ \\
$i \in \mathbb{Z}_{+} \times \mathbb{Z}_{+} \times\{-\infty\}$ & 0 & $-2 x_{t}(2)$ & $2 x_{t}(2)$ \\
Otherwise & Arbitrary & Arbitrary & Arbitrary \\
\hline
\end{tabular}

\subsection{ODE representation of limit trajectories}

This section establishes an explicit representation in terms of ordinary differential equations for the solutions of (2.4)-(2.7). Let $x$ denote such a solution. We start with a representation of the dynamics of $x$, which is based on (2.3). In the subsequent discussion $t$ is called a regular point of a function $g$ if $g$ is differentiable at $t$, and $\dot{g}_{t}$ denotes the derivative of $g$ at a regular point $t$.

Lemma 2.2. If $x$ satisfies (2.5)-(2.7) then it is differentiable at almost all $t \geq 0$. For almost all regular points $t$ of $x, \dot{x}_{t}=\sum_{i \in E} p_{x_{t}}(i) \dot{x}_{t}^{i}$, where $\dot{x}_{t}^{i}$ can be taken as in Table 1 .

Proof. If $x$ satisfies (2.5)-(2.7) then it is absolutely continuous, hence differentiable at almost all $t \geq 0$. For such $t,(2.3)$ implies that $\dot{x}_{t}=\sum_{i \in E} p_{x_{t}}(i) \dot{x}_{t}^{i}$, where $\dot{x}_{t}^{i}$ satisfies the following equations:

$$
\begin{aligned}
& \dot{x}_{t}^{i}(1)=\lambda_{\mathrm{f}} \pi_{x_{t}}^{i}\left(A_{1}\right)-x_{t}(1) \\
& \dot{x}_{t}^{i}(2)=\lambda_{\mathrm{h}} \pi_{x_{t}}^{i}\left(A_{2}\right)-2 x_{t}(2) \\
& \dot{x}_{t}^{i}(3)=\lambda_{\mathrm{h}} \pi_{x_{t}}^{i}\left(A_{3}\right)+2 x_{t}(2)-\lambda_{\mathrm{h}} \pi_{x_{t}}^{i}\left(A_{2}\right)-x_{t}(3) .
\end{aligned}
$$

To complete the proof it suffices to obtain the probabilities $\pi_{x_{t}}^{i}\left(A_{1}\right), \pi_{x_{t}}^{i}\left(A_{2}\right)$, and $\pi_{x_{t}}^{i}\left(A_{3}\right)$ in the case when the process $Y^{x_{t}, i}$ is ergodic. We consider each row of Table 1 separately.

- $i=(+\infty,+\infty,+\infty): Y^{x_{t}, i}$ is ergodic and $\left(\pi_{x_{t}}^{i}\left(A_{1}\right), \pi_{x_{t}}^{i}\left(A_{2}\right), \pi_{x_{t}}^{i}\left(A_{3}\right)\right)=(1,1,0)$.

- $i \in\{+\infty\} \times\{+\infty\} \times \mathbb{Z}$ : One may appeal to Figure 2(b) to see that $Y^{x_{t}, i}(3)$ is a homogeneous jump process on $\mathbb{Z}$. In particular $Y^{x_{t}, i}(3)$, and hence $Y^{x_{t}, i}$, is not ergodic and in turn $\pi_{x_{t}}^{i}$ can be chosen arbitrarily.

- $i=(+\infty,+\infty,-\infty): Y^{x_{t}, i}$ is ergodic and $\left(\pi_{x_{t}}^{i}\left(A_{1}\right), \pi_{x_{t}}^{i}\left(A_{2}\right), \pi_{x_{t}}^{i}\left(A_{3}\right)\right)=(1,0,0)$.

- $i \in\{+\infty\} \times \mathbb{Z}_{+} \times\{-\infty\}$ : If $Y^{x_{t}, i}$ is ergodic then the long-term rate of down-jumps of $Y^{x_{t}, i}(2), \lambda_{\mathrm{f}} \pi_{x_{t}}^{i}\left(A_{1}\right)$, is necessarily equal to the long-term rate of up-jumps of $Y^{x_{t}, i}(2)$, $x_{t}(1)+x_{t}(3)$. In particular $\left(\pi_{x_{t}}^{i}\left(A_{1}\right), \pi_{x_{t}}^{i}\left(A_{2}\right), \pi_{x_{t}}^{i}\left(A_{3}\right)\right)=\left(\left(x_{t}(1)+x_{t}(3)\right) / \lambda_{\mathrm{f}}, 0,0\right)$.

If $i(1) \in \mathbb{Z}_{+}$then it is enough to consider the case when $x_{t}(3)=0$, since otherwise Lemma 2.1 implies that $p_{x_{t}}(i)=0$, and thus $\pi_{x_{t}}^{i}$ can be chosen arbitrarily. 
- $i \in \mathbb{Z}_{+} \times\{+\infty\} \times\{+\infty\}$ : If $Y^{x_{t}, i}$ is ergodic then $\lambda_{\mathrm{h}} \pi_{x_{t}}^{i}\left(A_{2}\right)=2 x_{t}(2)+\lambda_{\mathrm{h}} \pi_{x_{t}}^{i}\left(A_{3}\right)$ and $\pi_{x_{t}}^{i}\left(A_{3}\right)=1-\pi_{x_{t}}^{i}\left(A_{2}\right)$; therefore $\left(\pi_{x_{t}}^{i}\left(A_{1}\right), \pi_{x_{t}}^{i}\left(A_{2}\right), \pi_{x_{t}}^{i}\left(A_{3}\right)\right)=\left(1,\left(\lambda_{\mathrm{h}}+2 x_{t}(2)\right) / 2 \lambda_{\mathrm{h}}\right.$, $\left.\left(\lambda_{\mathrm{h}}-2 x_{t}(2)\right) / 2 \lambda_{\mathrm{h}}\right)$.

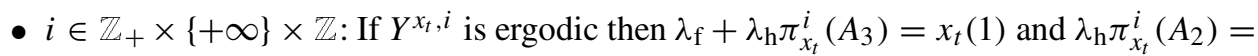
$2 x_{t}(2)+\lambda_{\mathrm{h}} \pi_{x_{t}}^{i}\left(A_{3}\right)$; therefore $\left(\pi_{x_{t}}^{i}\left(A_{1}\right), \pi_{x_{t}}^{i}\left(A_{2}\right), \pi_{x_{t}}^{i}\left(A_{3}\right)\right)=\left(1,\left(x_{t}(1)+2 x_{t}(2)-\right.\right.$ $\left.\left.\lambda_{\mathrm{f}}\right) / \lambda_{\mathrm{h}},\left(x_{t}(1)-\lambda_{\mathrm{f}}\right) / \lambda_{\mathrm{h}}\right)$.

- $i \in \mathbb{Z}_{+} \times\{+\infty\} \times\{-\infty\}$ : If $Y^{x_{t}, i}$ is ergodic then $\left(\pi_{x_{t}}^{i}\left(A_{1}\right), \pi_{x_{t}}^{i}\left(A_{2}\right), \pi_{x_{t}}^{i}\left(A_{3}\right)\right)=$ $(1,0,0)$.

- $i \in \mathbb{Z}_{+} \times \mathbb{Z}_{+} \times\{-\infty\}:$ If $Y^{x_{t}, i}$ is ergodic then $\lambda_{\mathrm{f}} \pi_{x_{t}}^{i}\left(A_{1}\right)=x_{t}(1)$, and therefore $\left(\pi_{x_{t}}^{i}\left(A_{1}\right), \pi_{x_{t}}^{i}\left(A_{2}\right), \pi_{x_{t}}^{i}\left(A_{3}\right)\right)=\left(x_{t}(1) / \lambda_{\mathrm{f}}, 0,0\right)$.

By Lemma $2.1 p_{x_{t}}(i)=0$ for the remaining values of $i \in E$; therefore $\pi_{x_{t}}^{i}$ can be chosen arbitrarily. The proof is completed by substituting the expressions obtained for $\pi_{x_{t}}^{i}\left(A_{1}\right)$, $\pi_{x_{t}}^{i}\left(A_{2}\right)$, and $\pi_{x_{t}}^{i}\left(A_{3}\right)$ in (2.8)-(2.10).

Define the sets $S_{1}=\{z \in S: z(3)=0\}, S_{2}=\{z \in S: z(1)+z(2)+z(3)=C\}$, and let $\overline{S_{1}}$ and $\overline{S_{2}}$ denote the respective complements. The following lemmas identify differential equations that govern the dynamics of the limit trajectory $x$ on four facets of the state space $S$ generated by $S_{1}$ and $S_{2}$. We now briefly outline the method of proof employed. In each proof the derivative $\dot{x}_{s}$ at a regular point $s$ is determined by first identifying the probability vector $p_{x_{s}}$, and then consulting Lemma 2.2. In seeking $p_{x_{s}}$, one first reduces the set of candidates by exploiting the fact that, depending on the point $x_{s}$, certain components of $p_{x_{s}}$ should vanish. Namely, by convention $p_{x_{s}}(i)=0$ if the process $Y^{x_{s}, i}$ is not ergodic, and by Lemma 2.1 $p_{x_{s}}(i)=0$ if at least one of the following conditions holds: (a) $i(2)<+\infty$ and $i(3)>-\infty$, (b) $i(1)<+\infty$ and $x_{s} \in \overline{S_{1}}$, and (c) $i(2) \wedge i(3)<+\infty$ and $x_{s} \in \overline{S_{2}}$. Note that some of the above conditions depend only on the facet that accommodates $x_{s}$. Finally, if $x$ spends nonzero time on $S_{1}$ or $S_{2}$, this places further requirements on $\dot{x}_{s}$ via the following remark, and consequently on $p_{x_{s}}$ via Lemma 2.2. It is then shown that these conditions determine essential features of $p_{x_{s}}$ so that $\dot{x}_{s}$ can be uniquely identified for almost all $s$.

The general argument of the following remark was used in a somewhat similar setting by Bean et al. (1997), and Hunt (1995).

Remark 2.1. For any absolutely continuous function $g$ and real number $c,\left\{t: g_{t}=c\right\} \subset$ $\left\{t: \dot{g}_{t}=0\right\} \cup N_{c}$ where $N_{c}$ is a set of zero Lebesgue measure. In particular $\dot{x}_{t}(3)=0$ for almost all $t \geq 0$ such that $x_{t} \in S_{1}$, and $\dot{x}_{t}(1)+\dot{x}_{t}(2)+\dot{x}_{t}(3)=0$ for almost all $t \geq 0$ such that $x_{t} \in S_{2}$.

Lemma 2.3. For almost all $t \geq 0$ such that $x_{t} \in \overline{S_{1}} \cap \overline{S_{2}}$,

$$
\dot{x}_{t}=\left(\lambda_{\mathrm{f}}-x_{t}(1), \lambda_{\mathrm{h}}-2 x_{t}(2), 2 x_{t}(2)-\lambda_{\mathrm{h}}-x_{t}(3)\right) .
$$

Proof. If $x_{t} \in \overline{S_{1}} \cap \overline{S_{2}}$ then $p_{x_{t}}(i)=\mathbf{1}\{i=(+\infty,+\infty,+\infty)\}, i \in E$. The lemma now follows by Lemma 2.2 which implies that

$$
\dot{x}_{t}=\dot{x}_{t}^{(+\infty,+\infty,+\infty)}=\left(\lambda_{\mathrm{f}}-x_{t}(1), \lambda_{\mathrm{h}}-2 x_{t}(2), 2 x_{t}(2)-\lambda_{\mathrm{h}}-x_{t}(3)\right) .
$$


Lemma 2.4. For almost all $t \geq 0$ such that $x_{t} \in S_{1} \cap \overline{S_{2}}$,

$$
x_{t}(2) \leq \lambda_{\mathrm{h}} / 2 \quad \text { and } \quad \dot{x}_{t}=\left(\lambda_{\mathrm{f}}-x_{t}(1),\left(\lambda_{\mathrm{h}} / 2\right)-x_{t}(2), 0\right) \text {. }
$$

Proof. By Remark 2.1 it suffices to establish the lemma for $t \geq 0$ such that $x_{t} \in S_{1} \cap \overline{S_{2}}$ and $\dot{x}_{t}(3)=0$. Let $t$ satisfy these conditions, and note that $p_{x_{t}}(i)>0$ only if $i(2)=i(3)=+\infty$. Consider the following two cases.

(i) $x_{t}(2) \geq \lambda_{\mathrm{h}} / 2$ : The process $Y^{x_{t}, i}$ is not ergodic for $i \in \mathbb{Z}_{+} \times\{+\infty\} \times\{+\infty\}$; thus by Lemma $2.2, \dot{x}_{t}=\dot{x}_{t}^{(+\infty,+\infty,+\infty)}=\left(\lambda_{\mathrm{f}}-x_{t}(1), \lambda_{\mathrm{h}}-2 x_{t}(2), 2 x_{t}(2)-\lambda_{\mathrm{h}}\right)$, and the condition $\dot{x}_{t}(3)=0$ is satisfied only if $x_{t}(2)=\lambda_{\mathrm{h}} / 2$.

(ii) $x_{t}(2)<\lambda_{\mathrm{h}} / 2: Y^{x_{t},(0,+\infty,+\infty)}$ is ergodic, and by Lemma 2.2 the condition $\dot{x}_{t}(3)=0$ is satisfied only if $\dot{x}_{t}=\dot{x}_{t}^{(0,+\infty,+\infty)}=\left(\lambda_{\mathrm{f}}-x_{t}(1),\left(\lambda_{\mathrm{h}} / 2\right)-x_{t}(2), 0\right)$. This establishes the lemma.

Lemma 2.5. For almost all $t \geq 0$ such that $x_{t} \in \overline{S_{1}} \cap S_{2}$ one of the following two conditions holds:

(a) $x_{t}(1)+x_{t}(3)<\lambda_{\mathrm{f}}$ and $\dot{x}_{t}=\left(x_{t}(3),-2 x_{t}(2), 2 x_{t}(2)-x_{t}(3)\right)$

(b) $x_{t}(1)+x_{t}(3)=\lambda_{\mathrm{f}}, x_{t}(2) \leq \lambda_{\mathrm{h}} / 2$, and $\dot{x}_{t}=\left(\lambda_{\mathrm{f}}-x_{t}(1), 0,-x_{t}(3)\right)$.

Proof. By Remark 2.1 it suffices to establish the lemma for $t \geq 0$ such that $x_{t} \in \overline{S_{1}} \cap S_{2}$ and $\dot{x}_{t}(1)+\dot{x}_{t}(2)+\dot{x}_{t}(3)=0$. Let $t$ satisfy these conditions, and note that $p_{x_{t}}(i)>0$ only if $i(1)=+\infty$. Consider the following two cases.

(i) $x_{t}(1)+x_{t}(3)<\lambda_{\mathrm{f}}$ : The process $Y^{x_{t},(+\infty, 0,-\infty)}$ is ergodic, and by Lemma 2.2 the condition $\dot{x}_{t}(1)+\dot{x}_{t}(2)+\dot{x}_{t}(3)=0$ holds only if

$$
\dot{x}_{t}=\dot{x}_{t}^{(+\infty, 0,-\infty)}=\left(x_{t}(3),-2 x_{t}(2), 2 x_{t}(2)-x_{t}(3)\right) .
$$

(ii) $x_{t}(1)+x_{t}(3) \geq \lambda_{\mathrm{f}}: Y^{x_{t}, i}$ is not ergodic for $i \in\{+\infty\} \times \mathbb{Z}_{+} \times\{-\infty\}$. Lemma 2.2 now implies that $\dot{x}_{t}=q \dot{x}_{t}^{(+\infty,+\infty,+\infty)}+(1-q) \dot{x}_{t}^{(+\infty,+\infty,-\infty)}$ for some $q \in[0,1]$; in turn the condition $\dot{x}_{t}(1)+\dot{x}_{t}(2)+\dot{x}_{t}(3)=0$ holds only if $x_{t}(1)+x_{t}(3)=\lambda_{\mathrm{f}}$. Appealing to Remark 2.1 we may concentrate on the case when $\dot{x}_{t}(1)+\dot{x}_{t}(3)=0$, which requires that $x_{t}(2) \leq \lambda_{\mathrm{h}} / 2$ so that $q=2 x_{t}(2) / \lambda_{\mathrm{h}}$ and by Lemma $2.2, \dot{x}_{t}=\left(\lambda_{\mathrm{f}}-x_{t}(1), 0,-x_{t}(3)\right)$. This completes the proof.

The following lemma, which is instrumental for the proof of Lemma 2.7, is proved in the Appendix.

Lemma 2.6. If $x_{t}(3)=0, x_{t}(2)<\lambda_{\mathrm{h}} / 2$, and $i \in \mathbb{Z}_{+} \times\{+\infty\} \times \mathbb{Z}_{\text {then }}$ the process $Y^{x_{t}, i}$ is ergodic only if $x_{t}(1)+x_{t}(2) \leq \lambda_{\mathrm{f}}+\left(\lambda_{\mathrm{h}} / 2\right)$.

Lemma 2.7. For almost all $t \geq 0$ such that $x_{t} \in S_{1} \cap S_{2}$ one of the following two conditions holds:

(a) $x_{t}(1)<\lambda_{\mathrm{f}}, x_{t}(2)=0$, and $\dot{x}_{t}=(0,0,0)$

(b) $x_{t}(1) \geq \lambda_{\mathrm{f}}, x_{t}(2) \leq \lambda_{\mathrm{h}} / 2, x_{t}(1)+x_{t}(2) \leq \lambda_{\mathrm{f}}+\left(\lambda_{\mathrm{h}} / 2\right)$, and

$$
\dot{x}_{t}=\left(\lambda_{\mathrm{f}}-x_{t}(1), x_{t}(1)-\lambda_{\mathrm{f}}, 0\right) \text {. }
$$


Proof. By Remark 2.1 it suffices to establish the lemma for $t \geq 0$ such that $x_{t} \in S_{1} \cap S_{2}$ and $\dot{x}_{t}(1)+\dot{x}_{t}(2)=\dot{x}_{t}(3)=0$. Let $t$ satisfy these conditions, and consider the following two cases. (i) $x_{t}(1)<\lambda_{\mathrm{f}}$ : The process $Y^{x_{t},(+\infty, 0,-\infty)}$ is ergodic, whereas $Y^{x_{t}, i}$ is ergodic for $i \in \mathbb{Z}_{+} \times\{+\infty\} \times\{+\infty\}$ only if $x_{t}(2)<\lambda_{\mathrm{h}} / 2$, and is not ergodic for $i \in \mathbb{Z}_{+} \times\{+\infty\} \times \mathbb{Z}$. Also note that for each $z \in \mathbb{Z}_{+}$Table 1 indicates that $\dot{x}_{t}^{(z,+\infty,-\infty)}=\dot{x}_{t}^{(+\infty,+\infty,-\infty)}$ and $\dot{x}_{t}^{(z, 0,-\infty)}=\dot{x}_{t}^{(+\infty, 0,-\infty)}$. One may thus appeal to Lemma 2.2 to write

$$
\dot{x}_{t}=q(1) \dot{x}_{t}^{(+\infty,+\infty,+\infty)}+q(2) \dot{x}_{t}^{(+\infty,+\infty,-\infty)}+q(3) \dot{x}_{t}^{(+\infty, 0,-\infty)}+q(4) \dot{x}_{t}^{(0,+\infty,+\infty)}
$$

for some probability vector $q \in \mathbb{R}_{+}^{4}$ such that $q(4)>0$ only if $x_{t}(2)<\lambda_{\mathrm{h}} / 2$. The condition $\dot{x}_{t}(1)+\dot{x}_{t}(2)+\dot{x}_{t}(3)=0$ is now satisfied only if $x_{t}(2)=0$ and $\dot{x}_{t}=\dot{x}_{t}^{(+\infty, 0,-\infty)}=(0,0,0)$.

(ii) $x_{t}(1) \geq \lambda_{\mathrm{f}}: Y^{x_{t}, i}$ is not ergodic if $i(2) \in \mathbb{Z}_{+}$; thus by Lemma $2.2 \dot{x}_{t}(1)=\lambda_{\mathrm{f}}-x_{t}(1)$, in turn the condition $\dot{x}_{t}(1)+\dot{x}_{t}(2)=\dot{x}_{t}(3)=0$ implies that $\dot{x}_{t}=\left(\lambda_{\mathrm{f}}-x_{t}(1), x_{t}(1)-\lambda_{\mathrm{f}}, 0\right)$. If $x_{t}(2) \geq \lambda_{\mathrm{h}} / 2$ then $Y^{x_{t}, i}$ is not ergodic if $i(1) \in \mathbb{Z}_{+}$, and by Lemma 2.2 it is necessary that $x_{t}(1)=\lambda_{\mathrm{f}}$ and $x_{t}(2)=\lambda_{\mathrm{h}} / 2$ so that $\dot{x}_{t}=\dot{x}_{t}^{(+\infty,+\infty,+\infty)}=(0,0,0)$. If $x_{t}(2)<\lambda_{\mathrm{h}} / 2$ then $Y^{x_{t},(0,+\infty,+\infty)}$ is ergodic, and Lemma 2.2 implies that

$$
\dot{x}_{t}=q(1) \dot{x}_{t}^{(+\infty,+\infty,+\infty)}+q(2) \dot{x}_{t}^{(+\infty,+\infty,-\infty)}+q(3) \dot{x}_{t}^{(0,+\infty,+\infty)}+q(4) \dot{x}_{t}^{(0,+\infty, 0)}
$$

for some probability vector $q \in \mathbb{R}_{+}^{4}$ such that $q(4)>0$ only if $Y^{x_{t},(0,+\infty, 0)}$ is ergodic. If $x_{t}(1)+x_{t}(2)>\lambda_{\mathrm{f}}+\left(\lambda_{\mathrm{h}} / 2\right)$ then by Lemma $2.6, Y^{x_{t},(0,+\infty, 0)}$ is not ergodic, and no such $q$ exists. Otherwise one can take

$$
\begin{array}{r}
q=\left(\left(1-\frac{2 x_{t}(2)}{\lambda_{\mathrm{h}}}\right)\left(1-\frac{x_{t}(1)-\lambda_{\mathrm{f}}}{\left(\lambda_{\mathrm{h}} / 2\right)-x_{t}(2)}\right),\left(\frac{2 x_{t}(2)}{\lambda_{\mathrm{h}}}\right)\left(1-\frac{x_{t}(1)-\lambda_{\mathrm{f}}}{\left(\lambda_{\mathrm{h}} / 2\right)-x_{t}(2)}\right),\right. \\
\left.\left(\frac{x_{t}(1)-\lambda_{\mathrm{f}}}{\left(\lambda_{\mathrm{h}} / 2\right)-x_{t}(2)}\right), 0\right) .
\end{array}
$$

This completes the proof.

Remark 2.2. The above proof indicates that the method employed here may not identify $\pi_{x_{t}}$ as a unique combination of distinct probability distributions. Such non-uniqueness arises elsewhere in the paper also (see the proof of Lemma 3.7), however the derivative $\dot{x}_{t}$ can still be uniquely identified in all cases.

\subsection{Proof of Theorem 1.1}

Lemmas $2.3,2.4,2.5$, and 2.7 can be shown to identify a unique limit trajectory issued from a given initial condition. Here we establish only the weaker claim that each limit trajectory converges to the point $x^{*} \in S$, which is an optimal operating point if $r_{\mathrm{f}} \geq 2 r_{\mathrm{h}}$. This implies that for large $\gamma$ the process $X^{\gamma}$ tends to remain in the vicinity of $x^{*}$, and leads to the proof of Theorem 1.1.

Lemma 2.8. If $x$ satisfies (2.4)-(2.7) then $\lim _{t \rightarrow \infty} x_{t}=x^{*}$ uniformly over initial conditions $x_{0} \in S$.

Proof. We prove the lemma by establishing the convergence of $x(1), x(3)$, and $x(2)$ in that order. Throughout the proof all limits are understood to be uniform in the initial condition.

Fix $\epsilon>0$. Lemmas $2.3,2.4,2.5$, and 2.7 imply that $\dot{x}_{t}(1) \geq\left(\lambda_{\mathrm{f}}-x_{t}(1)\right) \wedge x_{t}(3) \geq 0$ for almost all $t \geq 0$ such that $x_{t}(1)<x^{*}(1)-\epsilon$. For such $t$ either (i) $x_{t} \notin \overline{S_{1}} \cap S_{2}$ and $\dot{x}_{t}(1) \geq \epsilon$, or 
(ii) $x_{t} \in \overline{S_{1}} \cap S_{2}$ and either $\left(x_{t}(3) \geq \epsilon / 2\right.$ and $\left.\dot{x}_{t}(1) \geq \epsilon / 2\right)$ or $\left(x_{t}(3)<\epsilon / 2, \dot{x}_{t}(1)=x_{t}(3) \geq 0\right.$, and $\left.\ddot{x}_{t}(1)=2 x_{t}(2)-x_{t}(3)>\epsilon / 2\right)$. It thus follows that $x_{t}(1) \geq x^{*}(1)-\epsilon$ for all $t>1+(2 C / \epsilon)$, and the arbitrariness of $\epsilon$ yields that $\liminf _{t \rightarrow \infty} x_{t}(1) \geq x^{*}(1)$. Conditions (2.4) and (2.5) imply that $\limsup _{t \rightarrow \infty} x_{t}(1) \leq x^{*}(1)$; and consequently that $\lim _{t \rightarrow \infty} x_{t}(1)=x^{*}(1)$.

Appeal to the convergence of $x(1)$ and condition (2.6) to choose a $\tau(\epsilon)$ such that $x_{t}(1)>$ $x^{*}(1)-\epsilon$ and $x_{t}(2) \leq\left(\lambda_{\mathrm{h}} / 2\right)+(\epsilon / 4)$ for $t>\tau(\epsilon)$. For almost all $t>\tau(\epsilon)$ such that $x_{t}(3)>\epsilon$, Lemmas 2.5 and 2.3 imply that $x_{t} \in \overline{S_{1}} \cap \overline{S_{2}}$ and $\dot{x}_{t}(3)<-\epsilon / 2$ respectively. In particular $x_{t}(3)<\epsilon$ for all $t>\tau(\epsilon)+(2 C / \epsilon)$; thus $\lim _{t \rightarrow \infty} x_{t}(3)=0=x^{*}(3)$.

Appeal to the convergence of $x(1)$ and $x(3)$ to choose a $\tau^{\prime}(\epsilon)$ such that $x_{t}(1)<x^{*}(1)+\epsilon / 3$ and $x_{t}(3)<x^{*}(3)+\epsilon / 3$ for all $t>\tau^{\prime}(\epsilon)$. If $t$ satisfies these conditions and $x_{t}(2)<x^{*}(2)-\epsilon$ then $x_{t} \in \overline{S_{2}}$, and in turn Lemmas 2.3 and 2.4 imply that $\dot{x}_{t}(2)>\epsilon$ for almost all such $t$. Therefore $x_{t}(2) \geq x^{*}(2)-\epsilon$ for all $t>\tau^{\prime}(\epsilon)+C / \epsilon$, and $\liminf _{t \rightarrow \infty} x_{t}(2) \geq x^{*}(2)$. By conditions (2.4) and (2.6) $\lim \sup _{t \rightarrow \infty} x_{t}(2) \leq\left(C-x^{*}(1)-x^{*}(3)\right) \wedge\left(\lambda_{\mathrm{h}} / 2\right)=x^{*}(2)$; thus it follows that $\lim _{t \rightarrow \infty} x_{t}(2)=x^{*}(2)$. This completes the proof of the lemma.

Lemma 2.9. For any admission policy $\Pi$ and $\gamma>0$,

$$
J_{\Pi} / \gamma \leq \sup \left\{r_{\mathrm{f}} z(1)+r_{\mathrm{h}}(2 z(2)+z(3)): z \in S, z(1) \leq \lambda_{\mathrm{f}}, 2 z(2)+z(3) \leq \lambda_{\mathrm{h}}\right\} .
$$

Proof. Let $\epsilon>0$ be arbitrary, and set $H(\epsilon)=\left\{z \in \mathbb{R}_{+}^{3}: z \in S, z(1)<\lambda_{\mathrm{f}}+\epsilon, 2 z(2)+\right.$ $z(3)<\lambda_{\mathrm{h}}+\epsilon$. Since the process $X(1)$ (respectively the process $\left.2 X(2)+X(3)\right)$ is stochastically dominated by the number in an $M / M / \infty$ queue with load factor $\gamma \lambda_{\mathrm{f}}\left(\gamma \lambda_{\mathrm{h}}\right)$, there exists a $\tau(\epsilon)$ such that $\mathrm{E}\left[X_{t}^{\gamma}\right] \in H(\epsilon)$ for all $t>\tau(\epsilon)$. In turn

$$
\begin{aligned}
J_{\Pi} / \gamma & =\limsup _{T \rightarrow \infty} \frac{1}{T} \int_{0}^{T}\left\{r_{\mathrm{f}} \mathrm{E}\left[X_{t}^{\gamma}(1)\right]+r_{\mathrm{h}}\left(2 \mathrm{E}\left[X_{t}^{\gamma}(2)\right]+\mathrm{E}\left[X_{t}^{\gamma}(3)\right]\right)\right\} \mathrm{d} t \\
& \leq \sup \left\{r_{\mathrm{f}} z(1)+r_{\mathrm{h}}(2 z(2)+z(3)): z \in H(\epsilon)\right\} .
\end{aligned}
$$

The lemma follows by the arbitrariness of $\epsilon$.

Proof of Theorem 1.1. The claim that $\lim _{\gamma \rightarrow \infty} X_{\infty}^{\gamma}=x^{*}$ in probability follows by Lemma 2.8 and a direct adaptation of Lemma 7.2 of Alanyali and Hajek (1997). Since $\left(X_{\infty}^{\gamma}: \gamma>0\right)$ is uniformly integrable,

$$
\begin{aligned}
\lim _{\gamma \rightarrow \infty} J_{\mathrm{TRF}} / \gamma & =\lim _{\gamma \rightarrow \infty} \mathrm{E}\left[r_{\mathrm{f}} X_{\infty}^{\gamma}(1)+r_{\mathrm{h}}\left(2 X_{\infty}^{\gamma}(2)+X_{\infty}^{\gamma}(3)\right)\right] \\
& =\mathrm{E}\left[\lim _{\gamma \rightarrow \infty}\left\{r_{\mathrm{f}} X_{\infty}^{\gamma}(1)+r_{\mathrm{h}}\left(2 X_{\infty}^{\gamma}(2)+X_{\infty}^{\gamma}(3)\right)\right\}\right] \\
& =r_{\mathrm{f}} x^{*}(1)+r_{\mathrm{h}}\left(2 x^{*}(2)+x^{*}(3)\right) .
\end{aligned}
$$

Lemma 2.9, via straightforward minimization, yields that if $r_{\mathrm{f}} \geq 2 r_{\mathrm{h}}$ then any admission policy $\Pi$ satisfies $J_{\Pi} / \gamma \leq r_{\mathrm{f}} x^{*}(1)+r_{\mathrm{h}}\left(2 x^{*}(2)+x^{*}(3)\right)$ for all $\gamma>0$. This completes the proof.

\section{Trunk reservation for half-rate calls}

This section proves Theorem 1.2 by obtaining the limiting system dynamics, and thereby the asymptotic optimality of trunk reservation for half-rate calls in the case $r_{\mathrm{f}} \leq 2 r_{\mathrm{h}}$. The proof is obtained by streamlining the proof of Theorem 1.1. We start with establishing tightness of the sequence $\left(X^{\gamma}: \gamma>0\right)$, and the form of its weak limits along convergent subsequences. 

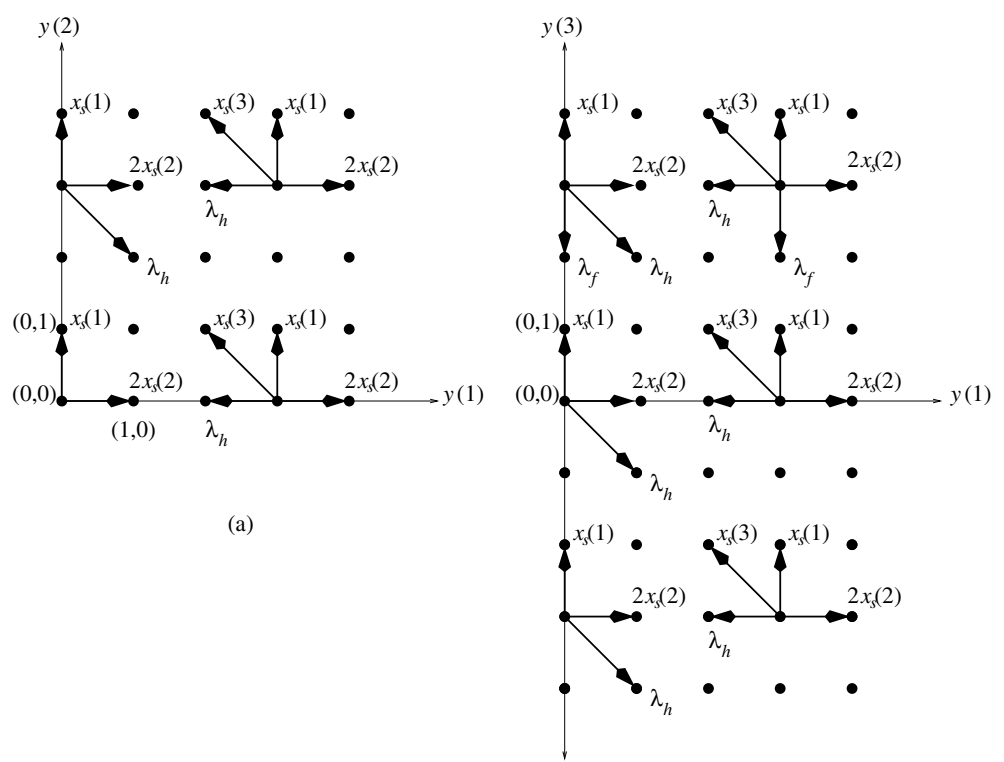

(b)

FIGURE 3: Transition diagrams of (a) the process $\left(Y^{x_{s}}(1), Y^{x_{s}}(2),-\infty\right)$ and (b) the process $\left(Y^{x_{s}}(1)\right.$, $\left.\infty, Y^{x_{s}}(3)\right)$, in the discussion of trunk reservation for half-rate calls.

Under trunk reservation for half-rate calls $X^{\gamma}$ is a Markov process on the state space $S$, and for $t \geq 0$

$$
\begin{aligned}
X_{t}^{\gamma}(1)= & X_{0}^{\gamma}(1)+\lambda_{\mathrm{f}} \int_{0}^{t} \mathbf{1}\left\{G_{s}>0\right\} \mathrm{d} s-\int_{0}^{t} X_{s}^{\gamma}(1) \mathrm{d} s+M_{t}^{\gamma}(1) \\
X_{t}^{\gamma}(2)= & X_{0}^{\gamma}(2)+\lambda_{\mathrm{h}} \int_{0}^{t} \mathbf{1}\left\{X_{s}(3)>0\right\} \mathrm{d} s-\int_{0}^{t} 2 X_{s}^{\gamma}(2) \mathrm{d} s+M_{t}^{\gamma}(2) \\
X_{t}^{\gamma}(3)= & X_{0}^{\gamma}(3)+\lambda_{\mathrm{h}} \int_{0}^{t} \mathbf{1}\left\{X_{s}(3)=0, F_{s}>0\right\} \mathrm{d} s+\int_{0}^{t} 2 X_{s}^{\gamma}(2) \mathrm{d} s \\
& -\lambda_{\mathrm{h}} \int_{0}^{t} \mathbf{1}\left\{X_{s}(3)>0\right\} \mathrm{d} s-\int_{0}^{t} X_{s}^{\gamma}(3) \mathrm{d} s+M_{t}^{\gamma}(3),
\end{aligned}
$$

where $M^{\gamma}=\left(M_{t}^{\gamma}: t \geq 0\right)$ is a martingale such that $M_{0}^{\gamma}=0$, and $\left(\left(F_{t}, G_{t}\right): t \geq 0\right)$ is defined as in Section 2. By redefining the sets $A_{1}, A_{2}, A_{3}$ as $A_{1}=\{y \in E: y(3)>0\}, A_{2}=$ $\{y \in E: y(1)>0\}$, and $A_{3}=\{y \in E: y(1)=0, y(2)>0\}$, the discussion of Section 2.1 applies verbatim and establishes that the sequence $\left(\left(X^{\gamma}, v^{\gamma}\right): \gamma>0\right)$ is tight. The limit $(x, v)$ of a weakly convergent subsequence of $\left(\left(X^{\gamma}, v^{\gamma}\right): \gamma>0\right)$ satisfies (2.4)-(2.7). Here $\pi_{x_{s}}$ is an equilibrium distribution of a Markov process $Y^{x_{s}}$ which takes values in $E$ and has transition rates given by (2.2). In effect $\left(Y^{x_{s}}(1), Y^{x_{s}}(2),-\infty\right)$ and $\left(Y^{x_{s}}(1), \infty, Y^{x_{s}}(3)\right)$ are two-dimensional Markov processes whose transition rates are specified by Figures 3(a) and 3(b) respectively, with the continuing understanding that $\pm \infty+k= \pm \infty$ for all $k \in \mathbb{Z}$.

Lemma 3.1. If $x$ satisfies (2.5)-(2.7) then it is differentiable at almost all $t \geq 0$. For almost all regular points $t$ of $x, \dot{x}_{t}=\sum_{i \in E} p_{x_{t}}(i) \dot{x}_{t}^{i}$, where $\dot{x}_{t}^{i}$ can be taken as in Table 2 . 
TABLE 2: Valid expressions for $\dot{x}_{t}^{i}$, under trunk reservation for half-rate calls.

\begin{tabular}{lccc}
\hline & $\dot{x}_{t}^{i}(1)$ & $\dot{x}_{t}^{i}(2)$ & $\dot{x}_{t}^{i}(3)$ \\
\hline$i=(+\infty,+\infty,+\infty)$ & $\lambda_{\mathrm{f}}-x_{t}(1)$ & $\lambda_{\mathrm{h}}-2 x_{t}(2)$ & $2 x_{t}(2)-\lambda_{\mathrm{h}}-x_{t}(3)$ \\
$i \in\{+\infty\} \times\{+\infty\} \times \mathbb{Z}$ & $x_{t}(3)$ & $\lambda_{\mathrm{h}}-2 x_{t}(2)$ & $2 x_{t}(2)-\lambda_{\mathrm{h}}-x_{t}(3)$ \\
$i=(+\infty,+\infty,-\infty)$ & $-x_{t}(1)$ & $\lambda_{\mathrm{h}}-2 x_{t}(2)$ & $2 x_{t}(2)-\lambda_{\mathrm{h}}-x_{t}(3)$ \\
$i \in\{+\infty\} \times \mathbb{Z}_{+} \times\{-\infty\}$ & $-x_{t}(1)$ & $\lambda_{\mathrm{h}}-2 x_{t}(2)$ & $2 x_{t}(2)-\lambda_{\mathrm{h}}-x_{t}(3)$ \\
$i \in \mathbb{Z}_{+} \times\{+\infty\} \times\{+\infty\}$ & $\lambda_{\mathrm{f}}-x_{t}(1)$ & $\left(\lambda_{\mathrm{h}} / 2\right)-x_{t}(2)$ & 0 \\
$i \in \mathbb{Z}_{+} \times\{+\infty\} \times \mathbb{Z}$ & $x_{t}(2)-\left(\lambda_{\mathrm{h}} / 2\right)$ & $\left(\lambda_{\mathrm{h}} / 2\right)-x_{t}(2)$ & 0 \\
$i \in \mathbb{Z}_{+} \times\{+\infty\} \times\{-\infty\}$ & $-x_{t}(1)$ & $\left(\lambda_{\mathrm{h}} / 2\right)-x_{t}(2)$ & 0 \\
$i \in \mathbb{Z}+\times \mathbb{Z}_{+} \times\{-\infty\}$ & $-x_{t}(1)$ & $x_{t}(1)$ & 0 \\
Otherwise & Arbitrary & Arbitrary & Arbitrary \\
\hline
\end{tabular}

Proof. If $x$ satisfies (2.5)-(2.7) then it is absolutely continuous, hence differentiable at almost all $t \geq 0$. For such $t$ the representation (2.3) implies that $\dot{x}_{t}=\sum_{i \in E} p_{x_{t}}(i) \dot{x}_{t}^{i}$, where $\dot{x}_{t}^{i}$ satisfies (2.8)-(2.10). The proof is completed by obtaining the probabilities $\pi_{x_{t}}^{i}\left(A_{1}\right), \pi_{x_{t}}^{i}\left(A_{2}\right)$, and $\pi_{x_{t}}^{i}\left(A_{3}\right)$ in the case when the process $Y^{x_{t}, i}$ is ergodic. We consider each row of Table 2 separately:

- $i=(+\infty,+\infty,+\infty): Y^{x_{t}, i}$ is ergodic and $\left(\pi_{x_{t}}^{i}\left(A_{1}\right), \pi_{x_{t}}^{i}\left(A_{2}\right), \pi_{x_{t}}^{i}\left(A_{3}\right)\right)=(1,1,0)$.

- $i \in\{+\infty\} \times\{+\infty\} \times \mathbb{Z}$ : If $Y^{x_{t}, i}$ is ergodic then $\lambda_{\mathrm{f}} \pi_{x_{t}}^{i}\left(A_{1}\right)=x_{t}(1)+x_{t}(3)$; therefore $\left(\pi_{x_{t}}^{i}\left(A_{1}\right), \pi_{x_{t}}^{i}\left(A_{2}\right), \pi_{x_{t}}^{i}\left(A_{3}\right)\right)=\left(\left(x_{t}(1)+x_{t}(3)\right) / \lambda_{\mathrm{f}}, 1,0\right)$.

- $i=(+\infty,+\infty,-\infty): Y^{x_{t}, i}$ is ergodic and $\left(\pi_{x_{t}}^{i}\left(A_{1}\right), \pi_{x_{t}}^{i}\left(A_{2}\right), \pi_{x_{t}}^{i}\left(A_{3}\right)\right)=(0,1,0)$.

- $i \in\{+\infty\} \times \mathbb{Z}_{+} \times\{-\infty\}$ : If $Y^{x_{t}, i}$ is ergodic then $\left(\pi_{x_{t}}^{i}\left(A_{1}\right), \pi_{x_{t}}^{i}\left(A_{2}\right), \pi_{x_{t}}^{i}\left(A_{3}\right)\right)=$ $(0,1,0)$.

If $i(1) \in \mathbb{Z}_{+}$then without loss of generality we consider the case $x_{t}(3)=0$.

- $i \in \mathbb{Z}_{+} \times\{+\infty\} \times\{+\infty\}$ : If $Y^{x_{t}, i}$ is ergodic then $\lambda_{\mathrm{h}} \pi_{x_{t}}^{i}\left(A_{2}\right)=2 x_{t}(2)+\lambda_{\mathrm{h}} \pi_{x_{t}}^{i}\left(A_{3}\right)$ and $\pi_{x_{t}}^{i}\left(A_{3}\right)=1-\pi_{x_{t}}^{i}\left(A_{2}\right)$; therefore

$$
\left(\pi_{x_{t}}^{i}\left(A_{1}\right), \pi_{x_{t}}^{i}\left(A_{2}\right), \pi_{x_{t}}^{i}\left(A_{3}\right)\right)=\left(1,\left(\lambda_{\mathrm{h}}+2 x_{t}(2)\right) / 2 \lambda_{\mathrm{h}},\left(\lambda_{\mathrm{h}}-2 x_{t}(2)\right) / 2 \lambda_{\mathrm{h}}\right) .
$$

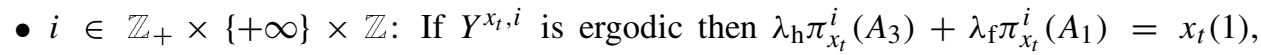
$\lambda_{\mathrm{h}} \pi_{x_{t}}^{i}\left(A_{2}\right)=\lambda_{\mathrm{h}} \pi_{x_{t}}^{i}\left(A_{3}\right)+2 x_{t}(2)$, and $\pi_{x_{t}}^{i}\left(A_{3}\right)=1-\pi_{x_{t}}^{i}\left(A_{2}\right)$; therefore

$$
\begin{aligned}
&\left(\pi_{x_{t}}^{i}\left(A_{1}\right), \pi_{x_{t}}^{i}\left(A_{2}\right), \pi_{x_{t}}^{i}\left(A_{3}\right)\right) \\
& \quad=\left(\left(x_{t}(1)+x_{t}(2)-\left(\lambda_{\mathrm{h}} / 2\right)\right) / \lambda_{\mathrm{f}},\left(\lambda_{\mathrm{h}}+2 x_{t}(2)\right) / 2 \lambda_{\mathrm{h}},\left(\lambda_{\mathrm{h}}-2 x_{t}(2)\right) / 2 \lambda_{\mathrm{h}}\right) .
\end{aligned}
$$

- $i \in \mathbb{Z}_{+} \times\{+\infty\} \times\{-\infty\}:$ If $Y^{x_{t}, i}$ is ergodic then $\lambda_{\mathrm{h}} \pi_{x_{t}}^{i}\left(A_{2}\right)=2 x_{t}(2)+\lambda_{\mathrm{h}} \pi_{x_{t}}^{i}\left(A_{3}\right)$ and $\pi_{x_{t}}^{i}\left(A_{3}\right)=1-\pi_{x_{t}}^{i}\left(A_{2}\right)$; therefore

$$
\left(\pi_{x_{t}}^{i}\left(A_{1}\right), \pi_{x_{t}}^{i}\left(A_{2}\right), \pi_{x_{t}}^{i}\left(A_{3}\right)\right)=\left(0,\left(\lambda_{\mathrm{h}}+2 x_{t}(2)\right) / 2 \lambda_{\mathrm{h}},\left(\lambda_{\mathrm{h}}-2 x_{t}(2)\right) / 2 \lambda_{\mathrm{h}}\right) .
$$


- $i \in \mathbb{Z}_{+} \times \mathbb{Z}_{+} \times\{-\infty\}$ : If $Y^{x_{t}, i}$ is ergodic then $\lambda_{\mathrm{h}} \pi_{x_{t}}^{i}\left(A_{2}\right)=\lambda_{\mathrm{h}} \pi_{x_{t}}^{i}\left(A_{3}\right)+2 x_{t}(2)$ and $\lambda_{\mathrm{h}} \pi_{x_{t}}^{i}\left(A_{3}\right)=x_{t}(1) ;$ therefore

$$
\left(\pi_{x_{t}}^{i}\left(A_{1}\right), \pi_{x_{t}}^{i}\left(A_{2}\right), \pi_{x_{t}}^{i}\left(A_{3}\right)\right)=\left(0,\left(x_{t}(1)+2 x_{t}(2)\right) / \lambda_{\mathrm{h}}, x_{t}(1) / \lambda_{\mathrm{h}}\right) .
$$

By Lemma $2.1 p_{x_{t}}(i)=0$ for the remaining values of $i \in E$; therefore $\pi_{x_{t}}^{i}$ can be chosen arbitrarily. The lemma now follows by substituting the expressions for probabilities $\pi_{x_{t}}^{i}\left(A_{1}\right)$, $\pi_{x_{t}}^{i}\left(A_{2}\right), \pi_{x_{t}}^{i}\left(A_{1}\right)$ in (2.8)-(2.10).

A trajectory $x$ satisfying (2.4)-(2.7) conforms to explicit differential equations as identified by the following lemmas. The proofs of Lemmas 3.2 and 3.3 are identical to the proofs of Lemmas 2.3 and 2.4 respectively, except that Lemma 2.2 is replaced by Lemma 3.1.

Lemma 3.2. For almost all $t \geq 0$ such that $x_{t} \in \overline{S_{1}} \cap \overline{S_{2}}$,

$$
\dot{x}_{t}=\left(\lambda_{\mathrm{f}}-x_{t}(1), \lambda_{\mathrm{h}}-2 x_{t}(2), 2 x_{t}(2)-\lambda_{\mathrm{h}}-x_{t}(3)\right) \text {. }
$$

Lemma 3.3. For almost all $t \geq 0$ such that $x_{t} \in S_{1} \cap \overline{S_{2}}$,

$$
x_{t}(2) \leq \lambda_{\mathrm{h}} / 2 \quad \text { and } \quad \dot{x}_{t}=\left(\lambda_{\mathrm{f}}-x_{t}(1),\left(\lambda_{\mathrm{h}} / 2\right)-x_{t}(2), 0\right) \text {. }
$$

Lemma 3.4. For almost all $t \geq 0$ such that $x_{t} \in \overline{S_{1}} \cap S_{2}$ one of the following two conditions holds:

(a) $x_{t}(1)+x_{t}(3)<\lambda_{\mathrm{f}}$ and $\dot{x}_{t}=\left(x_{t}(3), \lambda_{\mathrm{h}}-2 x_{t}(2), 2 x_{t}(2)-\lambda_{\mathrm{h}}-x_{t}(3)\right)$

(b) $x_{t}(1)+x_{t}(3)=\lambda_{\mathrm{f}}, x_{t}(2)=\lambda_{\mathrm{h}} / 2$, and $\dot{x}_{t}=\left(x_{t}(3), \lambda_{\mathrm{h}}-2 x_{t}(2), 2 x_{t}(2)-\lambda_{\mathrm{h}}-x_{t}(3)\right)$.

Proof. By Remark 2.1 it suffices to establish the lemma for $t \geq 0$ such that $x_{t} \in \overline{S_{1}} \cap S_{2}$ and $\dot{x}_{t}(1)+\dot{x}_{t}(2)+\dot{x}_{t}(3)=0$. Let $t$ satisfy these conditions, and note that $p_{x_{t}}(i)>0$ only if $i(1)=+\infty$. Lemma 3.1 implies that $\dot{x}_{t}(2)=\lambda_{\mathrm{h}}-2 x_{t}(2)$ and $\dot{x}_{t}(3)=2 x_{t}(2)-\lambda_{\mathrm{h}}-$ $x_{t}(3)$; therefore it is necessary that $\dot{x}_{t}(1)=x_{t}(3)$. If $x_{t}(1)+x_{t}(3)<\lambda_{\mathrm{f}}$, then the process $Y^{x_{t},(+\infty,+\infty, 0)}$ is ergodic and $\dot{x}_{t}=\dot{x}_{t}^{(+\infty,+\infty, 0)}$. If $x_{t}(1)+x_{t}(3) \geq \lambda_{\mathrm{f}}$, then $Y^{x_{t}, i}$ is not ergodic for any $i \in\{+\infty\} \times\{+\infty\} \times \mathbb{Z}$, and the condition $\dot{x}_{t}(1)+\dot{x}_{t}(2)+\dot{x}_{t}(3)=0$ holds only if $x_{t}(1)+x_{t}(3)=\lambda_{\mathrm{f}}$ and $\dot{x}_{t}=\dot{x}_{t}^{(+\infty,+\infty,+\infty)}$. By Remark 2.1 we may concentrate on the case when $\dot{x}_{t}(1)+\dot{x}_{t}(3)=0$, which, via Lemma 3.1, requires that $x_{t}(2)=\lambda_{\mathrm{h}} / 2$. This completes the proof.

The following two lemmas are used in the proof of Lemma 3.7, and they are proved in the Appendix.

Lemma 3.5. If $x_{t}(3)=0$ and $i \in \mathbb{Z}_{+} \times \mathbb{Z}_{+} \times\{-\infty\}$ then the process $Y^{x_{t}, i}$ is ergodic if and only if $x_{t}(1)+x_{t}(2)<\lambda_{\mathrm{h}} / 2$.

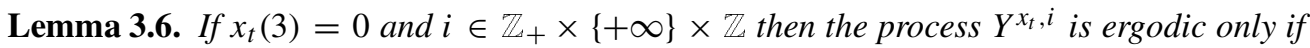
$\lambda_{\mathrm{h}} / 2 \leq x_{t}(1)+x_{t}(2) \leq \lambda_{\mathrm{f}}+\left(\lambda_{\mathrm{h}} / 2\right)$.

Lemma 3.7. For almost all $t \geq 0$ such that $x_{t} \in S_{1} \cap S_{2}$ one of the following three conditions holds:

(a) $x_{t}(2)=\lambda_{\mathrm{h}} / 2, x_{t}(1) \leq \lambda_{\mathrm{f}}$, and $\dot{x}_{t}=(0,0,0)$ 
(b) $x_{t}(1)+x_{t}(2)<\lambda_{\mathrm{h}} / 2$ and $\dot{x}_{t}=\left(-x_{t}(1), x_{t}(1), 0\right)$

(c) $x_{t}(2)<\lambda_{\mathrm{h}} / 2, \lambda_{\mathrm{h}} / 2 \leq x_{t}(1)+x_{t}(2) \leq \lambda_{\mathrm{f}}+\left(\lambda_{\mathrm{h}} / 2\right)$ and $\dot{x}_{t}=\left(x_{t}(2)-\left(\lambda_{\mathrm{h}} / 2\right),\left(\lambda_{\mathrm{h}} / 2\right)-\right.$ $\left.x_{t}(2), 0\right)$.

Proof. By Remark 2.1 it suffices to establish the lemma for $t \geq 0$ such that $x_{t} \in S_{1} \cap S_{2}$ and $\dot{x}_{t}(1)+\dot{x}_{t}(2)=\dot{x}_{t}(3)=0$. Let $t$ satisfy these conditions, and consider the following three cases.

(i) $x_{t}(2) \geq \lambda_{\mathrm{h}} / 2$ : Consult Lemmas 3.5 and 3.6 to see that the process $Y^{x_{t}, i}$ is not ergodic for any $i \in E$ such that $i(1) \in \mathbb{Z}_{+}$. Thus, by Lemma 3.1, the condition $\dot{x}_{t}(3)=0$ is satisfied only if $x_{t}(2)=\lambda_{\mathrm{h}} / 2$, in which case the condition $\dot{x}_{t}(1)+\dot{x}_{t}(2)=0$ is satisfied if either $x_{t}(1)=\lambda_{\mathrm{f}}$ and $\dot{x}_{t}=\dot{x}_{t}^{(+\infty,+\infty,+\infty)}$, or $x_{t}(1)<\lambda_{\mathrm{f}}$ (so that $Y^{x_{t},(+\infty,+\infty, 0)}$ is ergodic) and $\dot{x}_{t}=\dot{x}_{t}^{(+\infty,+\infty, 0)}$.

(ii) $x_{t}(2)<\lambda_{\mathrm{h}} / 2$ and $x_{t}(1)+x_{t}(2)<\lambda_{\mathrm{h}} / 2$ : By Lemma $3.5, Y^{x_{t},(0,0,-\infty)}$ is ergodic whereas by Lemma $3.6 Y^{x_{t}, i}$ is not ergodic for any $i \in \mathbb{Z}_{+} \times\{+\infty\} \times \mathbb{Z}$; Lemma 3.1 now implies that $\dot{x}_{t}(1)+\dot{x}_{t}(2)=\dot{x}_{t}(3)=0$ only if $\dot{x}_{t}=\dot{x}_{t}^{(0,0,-\infty)}$.

(iii) $x_{t}(2)<\lambda_{\mathrm{h}} / 2$ and $x_{t}(1)+x_{t}(2) \geq \lambda_{\mathrm{h}} / 2$ : By Lemma $3.5, Y^{x_{t}, i}$ is not ergodic for any $i \in \mathbb{Z}_{+} \times \mathbb{Z}_{+} \times\{-\infty\}$; in turn Lemma 3.1 implies that

$$
\dot{x}_{t}=q(1) \dot{x}_{t}^{(0,+\infty,+\infty)}+q(2) \dot{x}_{t}^{(0,+\infty, 0)}+q(3) \dot{x}_{t}^{(0,+\infty,-\infty)}=\dot{x}_{t}^{(0,+\infty, 0)}
$$

for some probability vector $q \in \mathbb{R}_{+}^{3}$ such that $q(2)>0$ only if $Y^{x_{t},(0,+\infty, 0)}$ is ergodic. If $x_{t}(1)+x_{t}(2)>\lambda_{\mathrm{f}}+\left(\lambda_{\mathrm{h}} / 2\right)$ then Lemma 3.6 implies that $Y^{x_{t},(0,+\infty, 0)}$ is not ergodic, and no such $q$ exists. Otherwise one can choose $q=\left(\left(x_{t}(1)+x_{t}(2)-\left(\lambda_{\mathrm{h}} / 2\right)\right) / \lambda_{\mathrm{f}}\right), 0,\left(1-\left(\left(x_{t}(1)+\right.\right.\right.$ $\left.\left.\left.x_{t}(2)-\left(\lambda_{\mathrm{h}} / 2\right)\right) / \lambda_{\mathrm{f}}\right)\right)$. This completes the proof.

The following lemma identifies a unique fixed point for the solutions of (2.4)-(2.7), and leads to the proof of Theorem 1.2.

Lemma 3.8. If $x$ satisfies (2.4)-(2.7) then $\lim _{t \rightarrow \infty} x_{t}=x_{*}$ uniformly over initial conditions $x_{0} \in S$.

Proof. The lemma is proved by establishing convergences of $x(2), x(3)$, and $x(1)$ in that order. Throughout the proof all limits are understood to be uniform in the initial condition.

Fix $\epsilon>0$. By Lemmas 3.2, 3.3, 3.4, and $3.7 \dot{x}_{t}(2)>\epsilon$ for almost all $t$ such that $x_{t}(2)<x_{*}(2)-\epsilon$; therefore $\liminf _{t \rightarrow \infty} x_{t}(2) \geq x_{*}$ (2). Conditions (2.4) and (2.6) imply that $\limsup _{t \rightarrow \infty} x_{t}(2) \leq x_{*}(2)$, and thus $\lim _{t \rightarrow \infty} x_{t}(2)=x_{*}(2)$.

Let $\tau(\epsilon)$ be such that $x_{t}(2) \leq\left(\lambda_{\mathrm{h}} / 2\right)+(\epsilon / 4)$ for all $t>\tau(\epsilon)$. For almost all $t>\tau(\epsilon)$ such that $x_{t}(3)>\epsilon$, Lemmas 3.2 and 3.4 imply that $\dot{x}_{t}(3)<-\epsilon / 2$. In particular $x_{t}(3)<\epsilon$ for all $t>\tau(\epsilon)+(2 C / \epsilon)$; and therefore $\lim _{t \rightarrow \infty} x_{t}(3)=0=x_{*}(3)$.

Appeal to the convergence of $x(2)$ and $x(3)$ to choose a $\tau^{\prime}(\epsilon)$ such that $x_{t}(2)<x_{*}(2)+\epsilon / 3$ and $x_{t}(3)<\epsilon / 3$ for all $t>\tau^{\prime}(\epsilon)$. If $t$ satisfies these conditions and $x_{t}(1)<x_{*}(1)-\epsilon$ then $x_{t} \in \overline{S_{2}}$, in turn Lemmas 3.2 and 3.3 imply that $\dot{x}_{t}(1)>\epsilon$ for almost all such $t$. Thus $\liminf _{t \rightarrow \infty} x_{t}(1) \geq x_{*}(1)$. Conditions (2.4) and (2.5) imply that $\limsup _{t \rightarrow \infty} x_{t}(1) \leq(C-$ $\left.x_{*}(2)-x_{*}(3)\right) \wedge \lambda_{\mathrm{f}}=x_{*}(1)$, and it follows that $\lim _{t \rightarrow \infty} x_{t}(1)=x_{*}(1)$. This completes the proof of the lemma.

Proof of Theorem 1.2. The claim that $\lim _{\gamma \rightarrow \infty} X_{\infty}^{\gamma}=x_{*}$ in probability follows by Lemma 3.8 and a direct adaptation of Lemma 7.2 of Alanyali and Hajek (1997). Since $\left(X_{\infty}^{\gamma}: \gamma>0\right)$ is uniformly integrable, 
TABLE 3: Valid expressions for $\dot{x}_{t}^{i}$, under complete sharing.

\begin{tabular}{lccc}
\hline & $\dot{x}_{t}^{i}(1)$ & $\dot{x}_{t}^{i}(2)$ & $\dot{x}_{t}^{i}(3)$ \\
\hline$i \in\{+\infty\} \times\{+\infty\} \times \mathbb{Z}^{\Delta}$ & $\lambda_{\mathrm{f}}-x_{t}(1)$ & $\lambda_{\mathrm{h}}-2 x_{t}(2)$ & $2 x_{t}(2)-\lambda_{\mathrm{h}}-x_{t}(3)$ \\
$i \in\{+\infty\} \times \mathbb{Z}_{+} \times \mathbb{Z}_{\Delta}^{\Delta}$ & $x_{t}(3)$ & $\lambda_{\mathrm{h}}-2 x_{t}(2)$ & $2 x_{t}(2)-\lambda_{\mathrm{h}}-x_{t}(3)$ \\
$i \in \mathbb{Z}_{+} \times\{+\infty\} \times \mathbb{Z}_{\Delta} \Delta$ & $\lambda_{\mathrm{f}}-x_{t}(1)$ & $\left(\lambda_{\mathrm{h}} / 2\right)-x_{t}(2)$ & 0 \\
$i \in \mathbb{Z}_{+} \times \mathbb{Z}_{+} \times \mathbb{Z}^{\Delta}$ & $-\lambda_{\mathrm{h}} \pi_{x_{t}}^{i}\left(A_{3}\right)$ & $\lambda_{\mathrm{h}} \pi_{x_{t}}^{i}\left(A_{3}\right)$ & 0 \\
\hline
\end{tabular}

$$
\begin{aligned}
\lim _{\gamma \rightarrow \infty} J_{\mathrm{TRH}} / \gamma & =\lim _{\gamma \rightarrow \infty} \mathrm{E}\left[r_{\mathrm{f}} X_{\infty}^{\gamma}(1)+r_{\mathrm{h}}\left(2 X_{\infty}^{\gamma}(2)+X_{\infty}^{\gamma}(3)\right)\right] \\
& =\mathrm{E}\left[\lim _{\gamma \rightarrow \infty}\left\{r_{\mathrm{f}} X_{\infty}^{\gamma}(1)+r_{\mathrm{h}}\left(2 X_{\infty}^{\gamma}(2)+X_{\infty}^{\gamma}(3)\right)\right\}\right] \\
& =r_{\mathrm{f}} x_{*}(1)+r_{\mathrm{h}}\left(2 x_{*}(2)+x_{*}(3)\right) .
\end{aligned}
$$

Lemma 2.9, via straightforward minimization, yields that if $r_{\mathrm{f}} \leq 2 r_{\mathrm{h}}$ then any admission policy $\Pi$ satisfies $J_{\Pi} / \gamma \leq r_{\mathrm{f}} x_{*}(1)+r_{\mathrm{h}}\left(2 x_{*}(2)+x_{*}(3)\right)$ for all $\gamma>0$. This completes the proof.

\section{Complete sharing}

This section proves Theorem 1.3 on the asymptotic optimality of complete sharing in the case $r_{\mathrm{f}} \leq 2 r_{\mathrm{h}}$. As in the previous section, the proof here is also obtained by streamlining the proof of Theorem 1.1.

Under complete sharing, $X^{\gamma}$ is a Markov process on the state space $S$ such that for $t \geq 0$

$$
\begin{aligned}
X_{t}^{\gamma}(1)= & X_{0}^{\gamma}(1)+\lambda_{\mathrm{f}} \int_{0}^{t} \mathbf{1}\left\{F_{s}>0\right\} \mathrm{d} s-\int_{0}^{t} X_{s}^{\gamma}(1) \mathrm{d} s+M_{t}^{\gamma}(1) \\
X_{t}^{\gamma}(2)= & X_{0}^{\gamma}(2)+\lambda_{\mathrm{h}} \int_{0}^{t} \mathbf{1}\left\{X_{s}(3)>0\right\} \mathrm{d} s-\int_{0}^{t} 2 X_{s}^{\gamma}(2) \mathrm{d} s+M_{t}^{\gamma}(2) \\
X_{t}^{\gamma}(3)= & X_{0}^{\gamma}(3)+\lambda_{\mathrm{h}} \int_{0}^{t} \mathbf{1}\left\{X_{s}(3)=0, F_{s}>0\right\} \mathrm{d} s+\int_{0}^{t} 2 X_{s}^{\gamma}(2) \mathrm{d} s \\
& -\lambda_{\mathrm{h}} \int_{0}^{t} \mathbf{1}\left\{X_{s}(3)>0\right\} \mathrm{d} s-\int_{0}^{t} X_{s}^{\gamma}(3) \mathrm{d} s+M_{t}^{\gamma}(3),
\end{aligned}
$$

where $M^{\gamma}=\left(M_{t}^{\gamma}: t \geq 0\right)$ is a martingale such that $M_{0}^{\gamma}=0$. By redefining the sets $A_{1}, A_{2}, A_{2}$ as $A_{1}=\{y \in E: y(2)>0\}, A_{2}=\{y \in E: y(1)>0\}$, and $A_{3}=\{y \in E:$ $y(1)=0, y(2)>0\}$, the discussion of Section 2.1 applies verbatim and establishes that the sequence $\left(\left(X^{\gamma}, v^{\gamma}\right): \gamma>0\right)$ is tight. The limit $(x, v)$ of a weakly convergent subsequence of $\left(\left(X^{\gamma}, v^{\gamma}\right): \gamma>0\right)$ satisfies (2.4)-(2.7). Here $\pi_{x_{s}}$ is an equilibrium distribution of a Markov process $Y^{x_{s}}$ which takes values in $E$ and has transition rates given by (2.2). In particular $\left(Y^{x_{s}}(1), Y^{x_{s}}(2)\right)$ is a Markov process whose transition rates are specified by Figure 4, with the continuing understanding that $\pm \infty+k= \pm \infty$ for all $k \in \mathbb{Z}$.

Lemma 4.1. If $x$ satisfies (2.5)-(2.7) then it is differentiable at almost all $t \geq 0$. For almost all regular points $t$ of $x, \dot{x}_{t}=\sum_{i \in E} p_{x_{t}}(i) \dot{x}_{t}^{i}$, where $\dot{x}_{t}^{i}$ can be taken as in Table 3.

Proof. If $x$ satisfies (2.5)-(2.7) then it is absolutely continuous, hence differentiable at almost all $t \geq 0$. For such $t$ the representation (2.3) implies that $\dot{x}_{t}=\sum_{i \in E} p_{x_{t}}(i) \dot{x}_{t}^{i}$, where $\dot{x}_{t}^{i}$ 


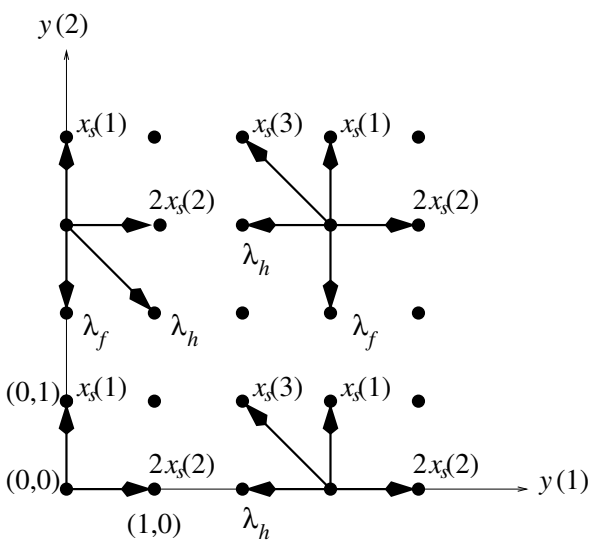

FIGURE 4: Transition diagram of the process $\left(Y^{x_{s}}(1), Y^{x_{s}}(2)\right)$, in the discussion of complete sharing. satisfies (2.8)-(2.10). We complete the proof by obtaining the probabilities $\pi_{x_{t}}^{i}\left(A_{1}\right), \pi_{x_{t}}^{i}\left(A_{2}\right)$, and $\pi_{x_{t}}^{i}\left(A_{3}\right)$ in the case when the process $Y^{x_{t}, i}$ is ergodic.

- $i \in\{+\infty\} \times\{+\infty\} \times \mathbb{Z}^{\Delta}: Y^{x_{t}, i}$ is ergodic and $\left(\pi_{x_{t}}^{i}\left(A_{1}\right), \pi_{x_{t}}^{i}\left(A_{2}\right), \pi_{x_{t}}^{i}\left(A_{3}\right)\right)=(1,1,0)$.

- $i \in\{+\infty\} \times \mathbb{Z}_{+} \times \mathbb{Z}^{\Delta}$ : If $Y^{x_{t}, i}$ is ergodic then $\lambda_{\mathrm{f}} \pi_{x_{t}}^{i}\left(A_{1}\right)=x_{t}(1)+x_{t}(3) ;$ therefore $\left(\pi_{x_{t}}^{i}\left(A_{1}\right), \pi_{x_{t}}^{i}\left(A_{2}\right), \pi_{x_{t}}^{i}\left(A_{3}\right)\right)=\left(\left(x_{t}(1)+x_{t}(3)\right) / \lambda_{\mathrm{f}}, 1,0\right)$.

If $i(1) \in \mathbb{Z}_{+}$then without loss of generality we consider the case $x_{t}(3)=0$.

- $i \in \mathbb{Z}_{+} \times\{+\infty\} \times \mathbb{Z}^{\Delta}$ : If $Y^{x_{t}, i}$ is ergodic then $\lambda_{\mathrm{h}} \pi_{x_{t}}^{i}\left(A_{2}\right)=2 x_{t}(2)+\lambda_{\mathrm{h}} \pi_{x_{t}}^{i}\left(A_{3}\right)$ and $\pi_{x_{t}}^{i}\left(A_{3}\right)=1-\pi_{x_{t}}^{i}\left(A_{2}\right)$; therefore $\left(\pi_{x_{t}}^{i}\left(A_{1}\right), \pi_{x_{t}}^{i}\left(A_{2}\right), \pi_{x_{t}}^{i}\left(A_{3}\right)\right)=\left(1,\left(\lambda_{\mathrm{h}}+2 x_{t}(2)\right) / 2 \lambda_{\mathrm{h}}\right.$, $\left.\left(\lambda_{\mathrm{h}}-2 x_{t}(2)\right) / 2 \lambda_{\mathrm{h}}\right)$.

- $i \in \mathbb{Z}_{+} \times \mathbb{Z}_{+} \times \mathbb{Z}^{\Delta}$ : If $Y^{x_{t}, i}$ is ergodic then $\lambda_{\mathrm{h}} \pi_{x_{t}}^{i}\left(A_{2}\right)=2 x_{t}(2)+\lambda_{\mathrm{h}} \pi_{x_{t}}^{i}\left(A_{3}\right)$ and $\lambda_{\mathrm{f}} \pi_{x_{t}}^{i}\left(A_{1}\right)+\lambda_{\mathrm{h}} \pi_{x_{t}}^{i}\left(A_{3}\right)=x_{t}(1) ;$ therefore

$$
\begin{aligned}
\left(\pi_{x_{t}}^{i}\left(A_{1}\right), \pi_{x_{t}}^{i}\left(A_{2}\right), \pi_{x_{t}}^{i}\left(A_{3}\right)\right) \\
=\left(\left(x_{t}(1)-\lambda_{\mathrm{h}} \pi_{x_{t}}^{i}\left(A_{3}\right)\right) / \lambda_{\mathrm{f}},\left(2 x_{t}(2)+\lambda_{\mathrm{h}} \pi_{x_{t}}^{i}\left(A_{3}\right)\right) / \lambda_{\mathrm{h}}, \pi_{x_{t}}^{i}\left(A_{3}\right)\right) .
\end{aligned}
$$

The lemma now follows by substituting the expressions for probabilities $\pi_{x_{t}}^{i}\left(A_{1}\right), \pi_{x_{t}}^{i}\left(A_{2}\right)$, $\pi_{x_{t}}^{i}\left(A_{1}\right)$ in (2.8)-(2.10).

A trajectory $x$ that satisfies (2.4)-(2.7) conforms to differential equations as identified by the following lemmas. The proofs of Lemmas 4.2 and 4.3 are identical to the proofs of Lemmas 2.3 and 2.4 respectively, except that Lemma 2.2 is replaced by Lemma 4.1.

Lemma 4.2. For almost all $t \geq 0$ such that $x_{t} \in \overline{S_{1}} \cap \overline{S_{2}}$,

$$
\dot{x}_{t}=\left(\lambda_{\mathrm{f}}-x_{t}(1), \lambda_{\mathrm{h}}-2 x_{t}(2), 2 x_{t}(2)-\lambda_{\mathrm{h}}-x_{t}(3)\right) \text {. }
$$

Lemma 4.3. For almost all $t \geq 0$ such that $x_{t} \in S_{1} \cap \overline{S_{2}}$,

$$
x_{t}(2) \leq \lambda_{\mathrm{h}} / 2 \quad \text { and } \quad \dot{x}_{t}=\left(\lambda_{\mathrm{f}}-x_{t}(1),\left(\lambda_{\mathrm{h}} / 2\right)-x_{t}(2), 0\right) \text {. }
$$


Lemma 4.4. For almost all $t \geq 0$ such that $x_{t} \in \overline{S_{1}} \cap S_{2}$ one of the following two conditions holds:

(a) $x_{t}(1)+x_{t}(3)<\lambda_{\mathrm{f}}$ and $\dot{x}_{t}=\left(x_{t}(3), \lambda_{\mathrm{h}}-2 x_{t}(2), 2 x_{t}(2)-\lambda_{\mathrm{h}}-x_{t}(3)\right)$

(b) $x_{t}(1)+x_{t}(3)=\lambda_{\mathrm{f}}, x_{t}(2)=\lambda_{\mathrm{h}} / 2$, and $\dot{x}_{t}=\left(x_{t}(3), \lambda_{\mathrm{h}}-2 x_{t}(2), 2 x_{t}(2)-\lambda_{\mathrm{h}}-x_{t}(3)\right)$.

Proof. By Remark 2.1 it suffices to establish the lemma for $t \geq 0$ such that $x_{t} \in \overline{S_{1}} \cap S_{2}$ and $\dot{x}_{t}(1)+\dot{x}_{t}(2)+\dot{x}_{t}(3)=0$. Let $t$ satisfy these conditions, and note that $p_{x_{t}}(i)>0$ only if $i(1)=+\infty$. Lemma 4.1 implies that $\dot{x}_{t}(2)=\lambda_{\mathrm{h}}-2 x_{t}(2)$ and $\dot{x}_{t}(3)=2 x_{t}(2)-\lambda_{\mathrm{h}}-$ $x_{t}(3)$; therefore it is necessary that $\dot{x}_{t}(1)=x_{t}(3)$. If $x_{t}(1)+x_{t}(3)<\lambda_{\mathrm{f}}$, then the process $Y^{x_{t},(+\infty, 0,-\infty)}$ is ergodic, and $\dot{x}_{t}=\dot{x}_{t}^{(+\infty, 0,-\infty)}$. If $x_{t}(1)+x_{t}(3) \geq \lambda_{\mathrm{f}}$, then $Y^{x_{t}, i}$ is not ergodic for any $i \in\{+\infty\} \times \mathbb{Z}_{+} \times\{-\infty\}$, and the condition $\dot{x}_{t}(1)+\dot{x}_{t}(2)+\dot{x}_{t}(3)=0$ holds only if $x_{t}(1)+x_{t}(3)=\lambda_{\mathrm{f}}$ and $\dot{x}_{t}=\dot{x}_{t}^{(+\infty,+\infty,+\infty)}$. By Remark 2.1 we may concentrate on the case when $\dot{x}_{t}(1)+\dot{x}_{t}(3)=0$, which, via Lemma 4.1 , requires that $x_{t}(2)=\lambda_{\mathrm{h}} / 2$. This completes the proof.

The following lemma is proved in the Appendix.

Lemma 4.5. If $x_{t}(3)=0$ and $i \in \mathbb{Z}_{+} \times \mathbb{Z}_{+} \times\{-\infty\}$ then the process $Y^{x_{t}, i}$ is ergodic if and only if $x_{t}(2)<\lambda_{\mathrm{h}} / 2$ and $x_{t}(1)+x_{t}(2)<\lambda_{\mathrm{f}}+\left(\lambda_{\mathrm{h}} / 2\right)$.

Lemma 4.6. For almost all $t \geq 0$ such that $x_{t} \in S_{1} \cap S_{2}$ one of the following three conditions holds:

(a) $x_{t}(2)=\lambda_{\mathrm{h}} / 2, x_{t}(1) \leq \lambda_{\mathrm{f}}$, and $\dot{x}_{t}=(0,0,0)$

(b) $x_{t}(2)<\lambda_{\mathrm{h}} / 2, x_{t}(1)+x_{t}(2)=\lambda_{\mathrm{f}}+\left(\lambda_{\mathrm{h}} / 2\right)$, and $\dot{x}_{t}=\left(\lambda_{\mathrm{f}}-x_{t}(1), \lambda_{\mathrm{h}}-2 x_{t}(2), 0\right)$

(c) $x_{t}(2)<\lambda_{\mathrm{h}} / 2, x_{t}(1)+x_{t}(2)<\lambda_{\mathrm{f}}+\left(\lambda_{\mathrm{h}} / 2\right)$, and

$$
\dot{x}_{t}=\left(-\lambda_{\mathrm{h}} \pi_{x_{t}}^{(0,0,-\infty)}\left(A_{3}\right), \lambda_{\mathrm{h}} \pi_{x_{t}}^{(0,0,-\infty)}\left(A_{3}\right), 0\right) .
$$

Proof. By Remark 2.1 it suffices to establish the lemma for $t \geq 0$ such that $x_{t} \in S_{1} \cap S_{2}$ and $\dot{x}_{t}(1)+\dot{x}_{t}(2)=\dot{x}_{t}(3)=0$. Let $t$ satisfy these conditions, and consider the following three cases.

(i) $x_{t}(2) \geq \lambda_{\mathrm{h}} / 2$ : Consult Lemma 4.5 to see that the process $Y^{x_{t}, i}$ is not ergodic for any $i \in E$ such that $i(1) \in \mathbb{Z}_{+}$. Thus by Lemma 4.1 the condition $\dot{x}_{t}(3)=0$ is satisfied only if $x_{t}(2)=\lambda_{\mathrm{h}} / 2$, in which case the condition $\dot{x}_{t}(1)+\dot{x}_{t}(2)=0$ is satisfied if either $x_{t}(1)=\lambda_{\mathrm{f}}$ and $\dot{x}_{t}=\dot{x}_{t}^{(+\infty,+\infty,+\infty)}$, or $x_{t}(1)<\lambda_{\mathrm{f}}$ (so that $Y^{x_{t},(+\infty, 0,-\infty)}$ is ergodic) and $\dot{x}_{t}=\dot{x}_{t}^{(+\infty, 0,-\infty)}$.

(ii) $x_{t}(2)<\lambda_{\mathrm{h}} / 2$ and $x_{t}(1)+x_{t}(2) \geq \lambda_{\mathrm{f}}+\left(\lambda_{\mathrm{h}} / 2\right): Y^{x_{t},(0,+\infty,+\infty)}$ is ergodic, whereas by Lemma $4.5 Y^{x_{t}, i}$ is not ergodic for $i \in \mathbb{Z}_{+} \times \mathbb{Z}_{+} \times\{-\infty\}$; therefore by Lemma 4.1, $\dot{x}_{t}(1)+\dot{x}_{t}(2)=\dot{x}_{t}(3)=0$ only if $x_{t}(1)+x_{t}(2)=\lambda_{\mathrm{f}}+\left(\lambda_{\mathrm{h}} / 2\right)$ and $\dot{x}_{t}=\dot{x}_{t}^{(0,+\infty,+\infty)}$.

(iii) $x_{t}(2)<\lambda_{\mathrm{h}} / 2$ and $x_{t}(1)+x_{t}(2)<\lambda_{\mathrm{f}}+\left(\lambda_{\mathrm{h}} / 2\right)$ : By Lemma $4.5, Y^{x_{t},(0,0,-\infty)}$ is ergodic, in turn by Lemma $4.1, \dot{x}_{t}=\dot{x}_{t}^{(0,0,-\infty)}$. This completes the proof.

We next establish a monotonicity property of the probability $\pi_{x_{t}}^{(0,0,-\infty)}\left(A_{3}\right)$, which is essential in identifying fixed points of limit trajectories. The proof of the following lemma can be found in the Appendix.

Lemma 4.7. If $C<\lambda_{\mathrm{f}}+\left(\lambda_{\mathrm{h}} / 2\right)$ then there exists a non-increasing function $h:\left[0, x_{*}(2)\right) \mapsto$ $(0,1]$ such that $h(\sigma) \leq \pi_{(C-\sigma, \sigma, 0)}^{(0,0,-\infty)}\left(A_{3}\right)$ for each $\sigma \in\left[0, x_{*}(2)\right)$. 
Lemma 4.8. If $x$ satisfies (2.4)-(2.7) then $\lim _{t \rightarrow \infty} x_{t}=x_{*}$ uniformly over initial conditions $x_{0} \in S$.

Proof. Fix $\epsilon>0$. For almost all $t$ such that $x_{t}(2)<x_{*}(2)-\epsilon$ either (i) $x_{t} \notin S_{1} \cap S_{2}$ and $\dot{x}_{t}(2)>\epsilon$ by Lemmas 4.2-4.4, or (ii) $x_{t} \in S_{1} \cap S_{2}$ and $\dot{x}_{t}(2)>\epsilon \wedge\left(\lambda_{\mathrm{h}} h\left(x_{*}(2)-\epsilon\right)\right)>0$ by Lemmas 4.6 and 4.7. In particular $\liminf _{t \rightarrow \infty} x_{t}(2) \geq x_{*}$ (2). Conditions (2.4) and (2.6) imply that $\lim \sup _{t \rightarrow \infty} x_{t}(2) \leq x_{*}(2)$; consequently $\lim _{t \rightarrow \infty} x_{t}(2)=x_{*}(2)$.

The proof of Lemma 3.8 now applies, with Lemmas 4.2 and 4.4 in place of Lemmas 2.3 and 2.5 respectively, to establish that $\lim _{t \rightarrow \infty} x_{t}(3)=x_{*}(3)$ and $\lim _{t \rightarrow \infty} x_{t}(1)=x_{*}(1)$. All limits are uniform in the initial condition, and the proof is complete.

Proof of Theorem 1.3. The proof of Theorem 1.2 applies by using Lemma 4.8 in place of Lemma 3.8.

\section{Appendix A.}

In this section we provide the proofs that are deferred in previous sections.

Proof of Lemma 2.1. Fix $K>0$ and let $B_{K}=\{y \in E: y(2)<y(3)+K\}$. Since $F_{t}=G_{t}+T(\gamma)$ for $t \geq 0$ and $\lim _{\gamma \rightarrow \infty} T(\gamma)=\infty$, definition (2.1) implies that $\nu^{\gamma}([0, \infty) \times$ $\left.B_{K}\right)=0$ for all large enough $\gamma$. Therefore $v\left([0, \infty) \times B_{K}\right)=0$, and by Lemma 5.8 of Royden (1988) the set $N_{K}=\left\{t \geq 0: \pi_{x_{t}}\left(B_{K}\right)>0\right\}$ has zero Lebesgue measure. The arbitrariness of $K$ now implies (a).

Fix $K, T, \delta>0$ and define $H_{T, \delta}=\left\{t \in[0, T): x_{t}(3)>\delta\right\}, B_{K}^{\prime}=\{y \in E: y(1)<K\}$. Since the convergence of $\left(X^{\gamma}: \gamma>0\right)$ is uniform on compact time sets, $v^{\gamma}\left(H_{T, \delta} \times B_{K}^{\prime}\right)$ converges to 0 in probability. In particular $v\left(H_{T, \delta} \times B_{K}^{\prime}\right)=0$, thus the set $N_{K, T, \delta}=\{t \in$ $\left.[0, T): x_{t}(3)>\delta, \pi_{x_{t}}\left(B_{K}^{\prime}\right)>0\right\}$ has zero Lebesgue measure. The arbitrariness of $K, T, \delta$ establishes (b).

Replacing the pair $(x(3), y(1))$ with $(C-(x(1)+x(2)+x(3)), y(2))$ in the above paragraph yields that $\pi_{x_{t}}(y(2)=+\infty)=1$ for almost all $t \geq 0$ such that $x_{t}(1)+x_{t}(2)+x_{t}(3)<C$. Since $\lim _{\gamma \rightarrow \infty} T(\gamma) / \gamma=0$, the same discussion applies when $y(2)$ is replaced by $y(3)$, thus it also follows that $\pi_{x_{t}}(y(3)=+\infty)=1$ for almost all such $t$. This establishes (c) and completes the proof.

The following remark is useful in several subsequent proofs.

Remark A.1. Let $U=\left(U_{k}: k \geq 0\right)$ denote the sequence of states visited by a Markov process $Y=\left(Y_{t}: t \geq 0\right)$ on a countable state space. By Theorem 2.1.2 of Asmussen (1987) $U$ is a Markov chain, and the transition probabilities of $U$ are proportional to the associated jump rates of $Y$. If $Y$ has bounded jump rates, then it follows from Theorem 2.4.3 of Asmussen (1987) that $Y$ is ergodic if and only if $U$ is positive recurrent.

Proof of Lemma 2.6. Let $U^{x_{t}, i}=\left(U_{k}^{x_{t}, i}: k \geq 0\right)$ denote the sequence of states visited by the process $Y^{x_{t}, i}$. By Remark A.1 it suffices to show that the chain $U^{x_{t}, i}$ is recurrent only if $x_{t}(1)+x_{t}(2) \leq \lambda_{\mathrm{f}}+\left(\lambda_{\mathrm{h}} / 2\right)$. We establish this via an adaptation of the methods of Zachary (1995), which concerns classification of Markov chains on $\mathbb{Z}_{+}^{2}$. Namely, if $x_{t}(1)+x_{t}(2)>\lambda_{\mathrm{f}}+$ $\left(\lambda_{\mathrm{h}} / 2\right)$ then Lemma 1 of Zachary (1995) implies existence of a negative function $g: \mathbb{Z}_{+} \mapsto \mathbb{R}$ and positive numbers $\epsilon, M$, such that the chain $\left(L_{k}=g\left(U_{k}^{x_{t}, i}(1)\right)+U_{k}^{x_{t}, i}(3): k \geq 0\right)$ satisfies

$$
\begin{gathered}
\mathrm{E}\left[L_{k+1}-L_{k} \mid U_{k}^{x_{t}, i}=u\right]>\epsilon \\
\mathrm{E}\left[\left|L_{k+1}-L_{k}\right|^{2} \mid U_{k}^{x_{t}, i}=u\right]<M,
\end{gathered}
$$




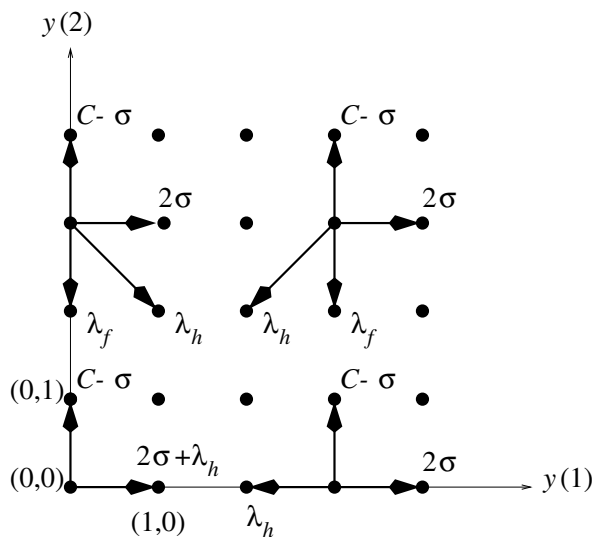

FIGURE 5: Transition diagram of the process $\left(W^{\sigma}(1), W^{\sigma}(2)\right)$.

for all $u \in \mathbb{Z}_{+} \times\{+\infty\} \times \mathbb{Z}_{\mathrm{S}}$ such that $g(u(1))+u(3)>0$. This follows by taking $\hat{P}$ as the transition probability matrix of $U^{x_{t},(0,+\infty,+\infty)}(1)$, and $\delta(\xi)=x_{t}(1)-\lambda_{\mathrm{f}}-\lambda_{\mathrm{h}} \mathbf{1}\{\xi=0\}$ for each $\xi \in \mathbb{Z}_{+}$, in Lemma 1 of Zachary (1995). Lemma 2 of Zachary (1995) now implies that $U^{x_{t}, i}$ is transient. This completes the proof.

Proof of Lemma 3.5. The lemma follows from Lemma 4.5 by taking $\lambda_{\mathrm{f}}=0$.

Proof of Lemma 3.6. If $Y^{x_{t}, i}$ is ergodic then it is necessary that $\lambda_{\mathrm{h}} \pi_{x_{t}}^{i}(y(1)>0)=$ $2 x_{t}(2)+\lambda_{\mathrm{h}} \pi_{x_{t}}^{i}(y(1)=0)$ and $x_{t}(1)=\lambda_{\mathrm{f}} \pi_{x_{t}}^{i}(y(3)>0)+\lambda_{\mathrm{h}} \pi_{x_{t}}^{i}(y(1)=0)$. In particular $\pi_{x_{t}}^{i}(y(3)>0)=\left(x_{t}(1)+x_{t}(2)-\left(\lambda_{\mathrm{h}} / 2\right)\right) / \lambda_{\mathrm{f}}$, which is a probability only if $\lambda_{\mathrm{h}} / 2 \leq x_{t}(1)+$ $x_{t}(2) \leq \lambda_{\mathrm{f}}+\left(\lambda_{\mathrm{h}} / 2\right)$. This establishes the lemma.

Proof of Lemma 4.5. Let $U^{x_{t}, i}=\left(U_{k}^{x_{t}, i}: k \geq 0\right)$ denote the sequence of states visited by the process $Y^{x_{t}, i}$. By Remark A.1 above and Theorems 3.3.1 and 3.4.1.(i) of Fayolle et al. (1995), $U^{x_{t}, i}$ is ergodic if and only if $x_{t}(2)<\lambda_{\mathrm{h}} / 2$ and $x_{t}(1)+x_{t}(2)<\lambda_{\mathrm{f}}+\left(\lambda_{\mathrm{h}} / 2\right)$. Since $Y^{x_{t}, i}$ has bounded jump rates the lemma follows.

The rest of this section proves Lemma 4.7. We start with some definitions that are relevant to the proof. Given $\sigma \in\left[0, x_{*}(2)\right)$ let $W^{\sigma}$ denote a Markov process which takes values in $E$, such that $W_{0}^{\sigma}=(0,0,-\infty)$ and $W^{\sigma}$ has transition rates given by

$$
W^{\sigma} \leftarrow \begin{cases}W^{\sigma}+(0,-1,-1) & \text { at rate } \lambda_{\mathrm{f}} \mathbf{1}\left\{W^{\sigma}(2)>0\right\} \\ W^{\sigma}+(0,+1,+1) & \text { at rate } C-\sigma \\ W^{\sigma}+(-1,0,0) & \text { at rate } \lambda_{\mathrm{h}} \mathbf{1}\left\{W^{\sigma}(1)>0, W^{\sigma}(2)=0\right\} \\ W^{\sigma}+(-1,-1,-1) & \text { at rate } \lambda_{\mathrm{h}} \mathbf{1}\left\{W^{\sigma}(1)>0, W^{\sigma}(2)>0\right\} \\ W^{\sigma}+(+1,0,0) & \text { at rate } 2 \sigma+\lambda_{\mathrm{h}} \mathbf{1}\left\{W^{\sigma}(1)=0, W^{\sigma}(2)=0\right\} \\ W^{\sigma}+(+1,-1,-1) & \text { at rate } \lambda_{\mathrm{h}} \mathbf{1}\left\{W^{\sigma}(1)=0, W^{\sigma}(2)>0\right\} .\end{cases}
$$

In particular $\left(W^{\sigma}(1), W^{\sigma}(2)\right)$ is a Markov process whose transition diagram is given by Figure 5 , and $W^{\sigma}(3) \equiv-\infty$. In the case when $W^{\sigma}$ is ergodic let $\mu^{\sigma}$ denote its equilibrium probability measure. Also let $A_{3}$ continue to denote the set $\{y \in E: y(1)=0, y(2)>0\}$.

Lemma A.1. If $C<\lambda_{\mathrm{f}}+\left(\lambda_{\mathrm{h}} / 2\right)$ and $\sigma \in\left[0, x_{*}(2)\right)$ then the process $W^{\sigma}$ is ergodic and $0<\mu^{\sigma}\left(A_{3}\right) \leq \pi_{(C-\sigma, \sigma, 0)}^{(0,0,-\infty)}\left(A_{3}\right)$. 
TABLE 4: Construction of the process $\left(W^{\sigma}, Y^{\sigma}\right)$.

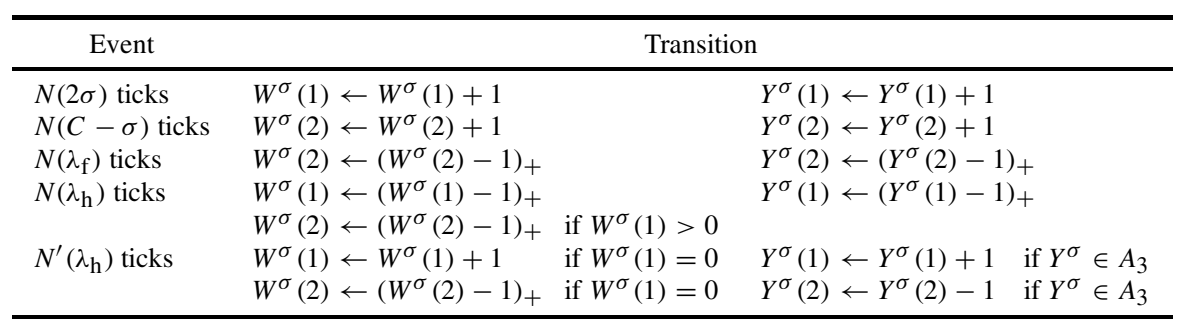

Proof. Let $C<\lambda_{\mathrm{f}}+\left(\lambda_{\mathrm{h}} / 2\right)$ and $\sigma \in\left[0, x_{*}(2)\right)$, and consult Remark A.1 and Theorem 3.3.1(a) of Fayolle et al. (1995) to see that $W^{\sigma}$ is ergodic. Since $A_{3}$ is an essential set for $W^{\sigma}, \mu^{\sigma}\left(A_{3}\right)>0$. The proof is completed by constructing a process $Y^{\sigma}$ on the same probability space as $W^{\sigma}$ so that $Y^{\sigma}$ is ergodic with equilibrium distribution $\pi_{(C-\sigma, \sigma, 0)}^{(0,0,-\infty)}$, and $Y_{t}^{\sigma} \in A_{3}$ for all $t$ such that $W_{t}^{\sigma} \in A_{3}$. Let $N(2 \sigma), N(C-\sigma), N\left(\lambda_{\mathrm{f}}\right), N\left(\lambda_{\mathrm{h}}\right)$, and $N^{\prime}\left(\lambda_{\mathrm{h}}\right)$ be independent Poisson clocks on an appropriate probability space, with respective rates $2 \sigma$, $C-\sigma, \lambda_{\mathrm{f}}, \lambda_{\mathrm{h}}$, and $\lambda_{\mathrm{h}}$. Set $W_{0}^{\sigma}=Y_{0}^{\sigma}=(0,0,-\infty)$ and construct the process $\left(W^{\sigma}, Y^{\sigma}\right)$ as indicated by Table 4 . Note that $Y^{\sigma}$ has the same distribution as $Y^{(C-\sigma, \sigma, 0),(0,0,-\infty)}$, and appeal to Lemma 4.5 to see that $Y^{\sigma}$ is ergodic.

Define $\tau=\inf \left\{t \geq 0: W_{t}^{\sigma}(1)<Y_{t}^{\sigma}(1)\right\}$. If $\tau$ is finite then by construction $W_{\tau^{-}}^{\sigma}(1)=$ $Y_{\tau^{-}}^{\sigma}(1)$, which implies that $W_{\tau}^{\sigma}(1) \geq Y_{\tau}^{\sigma}(1)$. This contradicts the definition of $\tau$; thus $\tau$ is infinite and

$$
W_{t}^{\sigma}(1) \geq Y_{t}^{\sigma}(1) \quad \text { for all } t \geq 0 \text {. }
$$

Suppose that $\eta=\inf \left\{t \geq 0: Y_{t}^{\sigma}(1)=0, W_{t}^{\sigma}(2)>Y_{t}^{\sigma}(2)\right\}$ is finite. Then the construction implies that $Y_{\eta^{-}}^{\sigma}(1)=1$ and

$$
N_{\eta}\left(\lambda_{\mathrm{h}}\right)=N_{\eta^{-}}\left(\lambda_{\mathrm{h}}\right)+1
$$

Set $\theta=\sup \left\{t<\eta: Y_{t}^{\sigma}(1)=0\right\}<\eta$. Since $Y_{\theta^{-}}^{\sigma}(1)=0$, the definition of $\eta$ implies that $W_{\theta^{-}}^{\sigma}(2) \leq Y_{\theta^{-}}^{\sigma}(2)$; in turn by construction $W_{\theta}^{\sigma}(2) \leq Y_{\theta}^{\sigma}(2)+1$. By observation (A.1), both $Y^{\sigma}(1)$ and $W^{\sigma}(1)$ are positive in the time interval $[\theta, \eta)$; therefore in this interval $Y^{\sigma}(2)$ increases every time $W^{\sigma}(2)$ increases, and $W^{\sigma}(2)$ decreases every time it is positive and $Y^{\sigma}(2)$ decreases. In particular $W_{\eta^{-}}^{\sigma}(2) \leq Y_{\eta^{-}}^{\sigma}(2)+1$. Observation (A.2) now implies that $W_{\eta}^{\sigma}(2) \leq$ $Y_{\eta}^{\sigma}(2)$. This contradicts the definition of $\eta$; thus $\eta$ is infinite. This and (A.1) imply that $Y_{t}^{\sigma} \in A_{3}$ for all $t$ such that $W_{t}^{\sigma} \in A_{3}$; therefore $\mu^{\sigma}\left(A_{3}\right) \leq \pi_{(C-\sigma, \sigma, 0)}^{(0,0,-\infty)}\left(A_{3}\right)$. This completes the proof.

Proof of Lemma 4.7. Let $0 \leq \sigma<\sigma^{\prime}<x_{*}(2)$, and let $N(2 \sigma), N\left(2\left(\sigma^{\prime}-\sigma\right)\right), N\left(C-\sigma^{\prime}\right)$, $N\left(\sigma^{\prime}-\sigma\right), N\left(\lambda_{\mathrm{f}}\right), N\left(\lambda_{\mathrm{h}}\right)$, and $N^{\prime}\left(\lambda_{\mathrm{h}}\right)$ denote independent Poisson clocks on an appropriate probability space, with respective rates $2 \sigma, 2\left(\sigma^{\prime}-\sigma\right), C-\sigma^{\prime}, \sigma^{\prime}-\sigma, \lambda_{\mathrm{f}}, \lambda_{\mathrm{h}}$, and $\lambda_{\mathrm{h}}$. Set $W_{0}^{\sigma}=W_{0}^{\sigma^{\prime}}=(0,0,-\infty)$ and construct the processes $W^{\sigma}$ and $W^{\sigma^{\prime}}$ on the same probability space as indicated by Table 5. Note that if $\tau=\inf \left\{t \geq 0: W_{t}^{\sigma^{\prime}}(1)<W_{t}^{\sigma}(1)\right\}$ is finite, then by construction $W_{\tau^{-}}^{\sigma^{\prime}}(1)=W_{\tau^{-}}^{\sigma}(1)$, which implies that $W_{\tau}^{\sigma^{\prime}}(1) \geq W_{\tau}^{\sigma}(1)$. This contradicts with the definition of $\tau$, therefore $\tau$ is infinite and $W_{t}^{\sigma^{\prime}}(1) \geq W_{t}^{\sigma}(1)$ for all $t$. Similarly, 
TABLE 5: Construction of the process $\left(W^{\sigma}, W^{\sigma^{\prime}}\right)$.

\begin{tabular}{|c|c|c|c|c|}
\hline Event & \multicolumn{4}{|c|}{ Transition } \\
\hline$N(2 \sigma)$ ticks & $W^{\sigma}(1) \leftarrow W^{\sigma}(1)+1$ & & $W^{\sigma^{\prime}}(1) \leftarrow W^{\sigma^{\prime}}(1)+1$ & \\
\hline$N\left(2\left(\sigma^{\prime}-\sigma\right)\right)$ ticks & - & & $W^{\sigma^{\prime}}(1) \leftarrow W^{\sigma^{\prime}}(1)+1$ & \\
\hline$N\left(C-\sigma^{\prime}\right)$ ticks & $W^{\sigma}(2) \leftarrow W^{\sigma}(2)+1$ & & $W^{\sigma^{\prime}}(2) \leftarrow W^{\sigma^{\prime}}(2)+1$ & \\
\hline$N\left(\sigma^{\prime}-\sigma\right)$ ticks & $W^{\sigma}(2) \leftarrow W^{\sigma}(2)+1$ & & - & \\
\hline$N\left(\lambda_{\mathrm{f}}\right)$ ticks & $W^{\sigma}(2) \leftarrow\left(W^{\sigma}(2)-1\right)_{+}$ & & $W^{\sigma^{\prime}}(2) \leftarrow\left(W^{\sigma^{\prime}}(2)-1\right)_{+}$ & \\
\hline$N\left(\lambda_{\mathrm{h}}\right)$ ticks & $\begin{array}{l}W^{\sigma}(1) \leftarrow\left(W^{\sigma}(1)-1\right)_{+} \\
W^{\sigma}(2) \leftarrow\left(W^{\sigma}(2)-1\right)\end{array}$ & if $W^{\sigma}(1)>0$ & $\begin{array}{l}W^{\sigma^{\prime}}(1) \leftarrow\left(W^{\sigma^{\prime}}(1)-1\right)_{+} \\
W^{\sigma^{\prime}}(2) \leftarrow\left(W^{\sigma^{\prime}}(2)-1\right)\end{array}$ & if $W^{\sigma^{\prime}}(1)>0$ \\
\hline$N^{\prime}\left(\lambda_{\mathrm{h}}\right)$ ticks & $\begin{array}{l}W^{\sigma}(1) \leftarrow W^{\sigma}(1)+1 \\
W^{\sigma}(2) \leftarrow\left(W^{\sigma}(2)-1\right)_{+}\end{array}$ & $\begin{array}{l}\text { if } W^{\sigma}(1)=0 \\
\text { if } W^{\sigma}(1)=0\end{array}$ & $\begin{array}{l}W^{\sigma^{\prime}}(1) \leftarrow W^{\sigma^{\prime}}(1)+1 \\
W^{\sigma^{\prime}}(2) \leftarrow\left(W^{\sigma^{\prime}}(2)-1\right)_{+}\end{array}$ & $\begin{array}{l}\text { if } W^{\sigma^{\prime}}(1)=0 \\
\text { if } W^{\sigma^{\prime}}(1)=0\end{array}$ \\
\hline
\end{tabular}

$W_{t}^{\sigma}(2) \geq W_{t}^{\sigma^{\prime}}(2)$ for all $t$. In particular $W_{t}^{\sigma} \in A_{3}$ for all $t$ such that $W_{t}^{\sigma^{\prime}} \in A_{3}$. Since $W^{\sigma}$ and $W^{\sigma^{\prime}}$ are ergodic by Lemma A.1, it follows that $\mu^{\sigma}\left(A_{3}\right) \geq \mu^{\sigma^{\prime}}\left(A_{3}\right)$. The lemma now follows by setting $h(\sigma)=\mu^{\sigma}\left(A_{3}\right)$, and appealing to Lemma A.1.

\section{Acknowledgement}

The author thanks a referee for carefully reading an earlier draft of the paper and for many valuable suggestions which improved the presentation.

\section{References}

Alanyali, M. And HajeK, B. (1997). Analysis of simple algorithms for dynamic load balancing. Math. Operat. Res. 22, 840-871.

Asmussen, S. (1987). Applied Probability and Queues. Wiley, Chichester.

Bean, N. G., Gibbens, R. J. And Zachary, S. (1995). Asymptotic analysis of single resource loss systems in heavy traffic, with applications to integrated networks. Adv. Appl. Prob. 27, 273-292.

Bean, N. G., Gibbens, R. J. And Zachary, S. (1997). Dynamic and equilibrium behaviour of controlled loss networks. Ann. Appl. Prob. 7, 873-885.

Ethier, S. N. AND Kurtz, T. G. (1986). Markov Processes: Characterization and Convergence. Wiley, New York.

Fayolle, G., Malyshev, V. A. And Menshikov, M. V. (1995). Topics in the Constructive Theory of Countable Markov Chains. Cambridge University Press, Cambridge.

Hunt, P. J. (1995). Pathological behaviour in loss networks. J. Appl. Prob. 32, 519-533.

Hunt, P. J. And Kurtz, T. G. (1994). Large loss networks. Stoch. Proc. Appl. 53, 363-378.

HUNT, P. J. AND LAWs, C. N. (1997). Optimization via trunk reservation in single resource loss systems under heavy traffic. Ann. Appl. Prob. 7, 1058-1079.

Kelly, F. P. (1986). Blocking probabilities in large circuit switched networks. Adv. Appl. Prob. 18, 473-505.

Miller, B. (1969). A queueing reward system with several customer classes. Management Sci. 16, $234-245$.

RAMASWAMI, V. AND RAO, K. A. (1985). Flexible time-slot assignment: a performance study for the integrated services digital network. Proc. ITC-11. Elsevier, New York, 2:2.1A-3-1-2.1A.3.7.

Reiman, M. I. AND SCHMitT, J. A. (1994). Performance models of multirate traffic in various network implementations. In Proc. ITC-14. Elsevier, New York, pp. 1217-1228.

Ross, K. W. AND TsAng, D. H. K. (1989). Optimal circuit access policies in an ISDN environment: a Markov decision approach. IEEE Trans. Commun. 37, 934-939.

Royden, H. L. (1988). Real Analysis. Macmillan, New York.

ZACHARY S. (1995). On two-dimensional Markov chains in the positive quadrant with partial spatial homogeneity. Markov Proc. Rel. Fields. 1, 267-280. 